\title{
\begin{tabular}{l|l} 
Mitraries & DSpace@MIT
\end{tabular}
}

\author{
MIT Open Access Articles
}

\section{ORBITAL DECAY OF HOT JUPITERS DUE TO NONLINEAR TIDAL DISSIPATION WITHIN SOLAR-TYPE HOSTS}

The MIT Faculty has made this article openly available. Please share how this access benefits you. Your story matters.

Citation: Essick, Reed, and Nevin N. Weinberg. “ORBITAL DECAY OF HOT JUPITERS DUE TO NONLINEAR TIDAL DISSIPATION WITHIN SOLAR-TYPE HOSTS.” The Astrophysical Journal 816, no. 1 (December 23, 2015): 18. (C) 2016 The American Astronomical Society

As Published: http://dx.doi.org/10.3847/0004-637x/816/1/18

Publisher: IOP Publishing

Persistent URL: http://hdl.handle.net/1721.1/101660

Version: Final published version: final published article, as it appeared in a journal, conference proceedings, or other formally published context

Terms of Use: Article is made available in accordance with the publisher's policy and may be subject to US copyright law. Please refer to the publisher's site for terms of use. 


\title{
ORBITAL DECAY OF HOT JUPITERS DUE TO NONLINEAR TIDAL DISSIPATION WITHIN SOLAR-TYPE HOSTS
}

\author{
Reed Essick And Nevin N. Weinberg \\ Department of Physics, and Kavli Institute for Astrophysics and Space Research, Massachusetts Institute of Technology, Cambridge, MA 02139, USA \\ Received 2015 August 17; accepted 2015 November 7; published 2015 December 23
}

\begin{abstract}
We study the orbital evolution of hot Jupiters due to the excitation and damping of tidally driven $g$-modes within solar-type host stars. Linearly resonant $g$-modes (the dynamical tide) are driven to such large amplitudes in the stellar core that they excite a sea of other $g$-modes through weakly nonlinear interactions. By solving the dynamics of large networks of nonlinearly coupled modes, we show that the nonlinear dissipation rate of the dynamical tide is several orders of magnitude larger than the linear dissipation rate. We find stellar tidal quality factors $Q_{*}^{\prime} \simeq 10^{5}$ $10^{6}$ for systems with planet mass $M_{p} \gtrsim 0.5 M_{\mathrm{J}}$ and orbital period $P \lesssim 2$ days, which implies that such systems decay on timescales that are small compared to the main-sequence lifetime of their solar-type hosts. According to our results, there are $\simeq 10$ currently known exoplanetary systems, including WASP-19b and HAT-P-36-b, with orbital decay timescales shorter than a Gyr. Rapid, tidally induced orbital decay may explain the observed paucity of planets with $M_{p} \gtrsim M_{\mathrm{J}}$ and $P<2$ days around solar-type hosts and could generate detectable transit-timing variations in the near future.
\end{abstract}

Key words: gravitation - instabilities - planets and satellites: dynamical evolution and stability - waves

\section{INTRODUCTION}

The tide raised by a hot Jupiter excites large amplitude waves within its host star. These waves transfer energy and angular momentum from the orbit to the star and as a result the planet gradually spirals inward. The rate of orbital decay is determined by the efficiency of tidal dissipation and depends on the amplitude of the waves as well as the effectiveness of frictional processes within the star.

Tidal dissipation is often parameterized by the stellar tidal quality factor $Q_{*}^{\prime}$, where larger $Q_{*}^{\prime}$ implies less dissipation. Perhaps the best constraints on $Q_{*}^{\prime}$ for solar-type stars come from the observed circularization rate of solar-type binaries, which yield $Q_{*}^{\prime} \sim 10^{6}$ (Meibom \& Mathieu 2005). However, because $Q_{*}^{\prime}$ is not a fundamental property of the star (it depends on the shape and size of the orbit and the mass of the perturber), this result does not necessarily imply $Q_{*}^{\prime} \sim 10^{6}$ for hot Jupiter systems. There have been a number of efforts to measure $Q_{*}^{\prime}$ from statistical modeling of the observed sample of hot Jupiters (see Ogilvie 2014 for a review). Penev et al. (2012) find that the distribution favors $Q_{*}^{\prime} \gtrsim 10^{7}$ for a specific set of assumptions about the initial conditions. Jackson et al. (2008) find a best fit at $Q_{*}^{\prime} \sim 10^{5.5}$ although they do not rule out much larger values and note that it is difficult to obtain tight constraints because of the limited sample size and uncertainties in the initial period distribution and stellar age. Although there are no direct observational measurements of $Q_{*}^{\prime}$ from individual hot Jupiter systems (e.g., from the detection of orbital decay), Jackson et al. (2009) argue that the distribution shows evidence for ongoing removal and destruction by tides. In addition, Teitler \& Königl (2014) propose that the observed dearth of close-in planets around fast-rotating stars (McQuillan et al. 2013) can be attributed to tidal ingestion of giant planets.

Linear tidal driving by the planet resonantly excites short wavelength waves within the host star. In solar-type stars, these "primary" waves are excited near the radiative-convective interface since in this region their wavelengths become large and they can couple to the long length scale tidal potential.
Although the primary waves initially have relatively small amplitudes and are thus well-described by linear theory, as they propagate toward the stellar center their amplitudes increase due to geometric focusing (i.e., in order to conserve WKB flux within an ever decreasing volume). In hot Jupiter systems, the primary waves reach large amplitudes as they approach the stellar core and become nonlinear, exciting many secondary waves through nonlinear wave-wave interactions (Barker \& Ogilvie 2010, 2011; Weinberg et al. 2012, hereafter WAQB). These secondary waves can have much shorter wavelengths than the primary waves and, as a result, they can have much larger damping rates (due to radiative diffusion). Systems in which nonlinear interactions are important may therefore dissipate tidal energy much more rapidly than the linear theory estimates. Indeed, in the case of solar-type binaries, the linear theory estimates yield dissipation rates that are too small by a factor of $\gtrsim 100\left(Q_{*}^{\prime} \sim 10^{8}-10^{10}\right.$; Goodman \& Dickson 1998; Terquem et al. 1998; Ogilvie \& Lin 2007). This may indicate that nonlinear processes are playing an important role in these systems.

For a planet with mass $M_{p} \gtrsim 3 M_{\mathrm{J}}(P / \text { day })^{-0.1}$ orbiting a solar-type star, the primary waves reach such large amplitudes near the stellar center that they overturn the background stratification and break (Barker \& Ogilvie 2010; Barker 2011). In this strongly nonlinear regime, the primary waves deposit nearly all of their energy and angular momentum in a single group travel time through the star. The tidal dissipation rate therefore equals the energy flux of the initial, linearly driven primary waves. The three-dimensional numerical simulations of wave breaking by Barker (2011) yield $Q_{*}^{\prime} \simeq 10^{5}(P / 1 \text { day })^{2.8}$ for $M_{p} \gtrsim 3 M_{\mathrm{J}}$ and a solar-type star. This corresponds to an inspiral time of $\approx 1 \mathrm{Gyr}$ for a $3 M_{\mathrm{J}}$ planet in a 2 day orbit.

For a planet with mass $0.5 \lesssim M_{p} / M_{\mathrm{J}} \lesssim 3$, the primary waves do not, in general, break. Nonetheless, they are sufficiently nonlinear that they excite many secondary waves near the stellar center. In this weakly nonlinear regime, the primary waves only deposit a fraction of their energy and angular 
momentum in a single group travel time. The value of that fraction, which determines the rate of tidal dissipation, depends on the detailed interaction between the primary waves and the sea of secondary waves. The aim of our study is to calculate this interaction (and its saturation) in the weakly nonlinear regime. Similar types of analyses have been carried out in the context of the $r$-mode instability in spinning neutron stars (Brink et al. 2005; Bondarescu et al. 2009).

This paper is structured as follows. In Section 2 we describe the formalism we use to study the weakly nonlinear tidal interactions and present the equations of motion for our mode decomposition. In Section 3 we describe how we construct our networks of interacting modes and our method for integrating the coupled equations of motion. In Section 4 we present a pedagogical discussion of how different mode networks behave. The main results of our calculations are presented in Section 5, with particular emphasis on the tidal evolution of known exoplanetary systems. Finally, in Section 6 we summarize our results and describe some of the limitations of our analysis that can serve as directions for future work.

\section{FORMALISM}

We are interested in calculating the orbital evolution of hot Jupiters due to tidal dissipation within the host star. We assume that the planet's orbit is circular, as is the case for most of the observed hot Jupiters (Udry \& Santos 2007; Ogilvie 2014). If the system is also sufficiently old so that the planet's rotation is synchronous with the orbit (Barker \& Lithwick 2014; Storch \& Lai 2014), then there is no tidal dissipation within the planet.

The tide raised by the planet excites a variety of oscillation modes within the star. Here we limit our analysis to solar-type hosts and focus on the excitation of resonant $g$-modes due to linear and (weakly) nonlinear forces. Because the orbital period of a hot Jupiter is much shorter than the rotational period of a solar-type star, the $g$-modes are not strongly modified by Coriolis forces and we therefore neglect the star's rotation.

\subsection{Equations of Motion}

We calculate the orbital evolution using the formalism developed in WAQB for studying tides in close binary systems in which weakly nonlinear wave interactions are important (see also Van Hoolst 1994; Schenk et al. 2001). We now briefly summarize the method and refer the reader to WAQB for a more detailed discussion.

The equation of motion for the Lagrangian displacement $\boldsymbol{\xi}(\boldsymbol{r}, t)$ of the stellar fluid at position $\boldsymbol{r}$ and time $t$ relative to the unperturbed background is

$$
\rho \ddot{\boldsymbol{\xi}}=\boldsymbol{f}_{1}[\boldsymbol{\xi}]+\boldsymbol{f}_{2}[\boldsymbol{\xi}, \boldsymbol{\xi}]+\rho \boldsymbol{a}_{\mathrm{tide}},
$$

where $\rho$ is the background density, $\boldsymbol{f}_{1}$ and $\boldsymbol{f}_{2}$ are the linear and leading-order nonlinear restoring forces,

$$
\boldsymbol{a}_{\text {tide }}=-\nabla U-(\xi \cdot \nabla) \nabla U
$$

is the tidal acceleration, and $U$ is the tidal potential. We include only the dominant $l=2$ tidal harmonic and since we assume that the orbit is circular,

$$
U(\boldsymbol{r}, t)=-\epsilon \omega_{0}^{2} r^{2} \sum_{m=-2}^{2} W_{2 m} Y_{2 m}(\theta, \phi) e^{-i m \Omega t},
$$

where $\epsilon=\left(M_{p} / M\right)(R / a)^{3}, \omega_{0}=\left(G M / R^{3}\right)^{1 / 2}$ is the dynamical frequency of a star with mass $M$ and radius $R, M_{p}$ is the planet mass, $a$ and $\Omega$ are the orbital semi-major axis and frequency, and $W_{20}=-(\pi / 5)^{1 / 2}, W_{2 \pm 2}=(3 \pi / 10)^{1 / 2}$, and $W_{2 \pm 1}=0$. We solve Equation (1) using the method of weighted residuals in which we expand the six-dimensional phase space vector as

$$
\left[\begin{array}{c}
\boldsymbol{\xi}(\boldsymbol{r}, t) \\
\partial_{t} \boldsymbol{\xi}(\boldsymbol{r}, t)
\end{array}\right]=\sum_{\alpha} q_{\alpha}(t)\left[\begin{array}{c}
\boldsymbol{\xi}_{\alpha}(\boldsymbol{r}) \\
i \omega_{\alpha} \boldsymbol{\xi}_{\alpha}(\boldsymbol{r})
\end{array}\right],
$$

where $\alpha$ labels a linear eigenmode with eigenfunction $\boldsymbol{\xi}_{\alpha}$, eigenfrequency $\omega_{\alpha}$, and amplitude $q_{\alpha}(t)$. The sum over $\alpha$ runs over all mode quantum numbers and frequency signs to allow both a mode and its complex conjugate. We normalize the eigenmodes as

$$
E_{0} \equiv \frac{G M^{2}}{R}=2 \omega_{\alpha}^{2} \int d^{3} x \rho \boldsymbol{\xi}_{\alpha}^{*} \cdot \boldsymbol{\xi}_{\alpha}
$$

so that a mode with dimensionless amplitude $\left|q_{\alpha}\right|=1$ has energy $E_{0}$. Plugging Equation (4) into (1), adding a linear damping term, and using the orthogonality of the eigenmodes leads to a coupled, nonlinear amplitude equation for each mode

$$
\begin{aligned}
& \dot{q}_{\alpha}+\left(i \omega_{\alpha}+\gamma_{\alpha}\right) q_{\alpha}= \\
& \quad i \omega_{\alpha}\left[U_{\alpha}(t)+\sum_{\beta} U_{\alpha \beta}^{*}(t) q_{\beta}^{*}+\sum_{\beta \gamma} \kappa_{\alpha \beta \gamma}^{*} q_{\beta}^{*} q_{\gamma}^{*}\right],
\end{aligned}
$$

where

$$
\begin{gathered}
U_{\alpha}(t)=-\frac{1}{E_{0}} \int d^{3} x \rho \boldsymbol{\xi}_{\alpha}^{*} \cdot \nabla U, \\
U_{\alpha \beta}(t)=-\frac{1}{E_{0}} \int d^{3} x \rho \boldsymbol{\xi}_{\alpha} \cdot\left(\boldsymbol{\xi}_{\beta} \cdot \nabla\right) \nabla U, \\
\kappa_{\alpha \beta \gamma}=\frac{1}{E_{0}} \int d^{3} x \boldsymbol{\xi}_{\alpha} \cdot \boldsymbol{f}_{2}\left[\boldsymbol{\xi}_{\beta}, \boldsymbol{\xi}_{\gamma}\right] .
\end{gathered}
$$

The coefficient $\gamma_{\alpha}$ is the linear damping rate of the mode, $U_{\alpha}$ and $U_{\alpha \beta}$ represent the linear and nonlinear tidal force, and $\kappa_{\alpha \beta \gamma}$ represents the three-mode coupling.

\subsection{Expressions for the Coefficients}

We consider the dynamics of high-order, adiabatic $g$-modes within a solar-type main-sequence star. These modes are restored by buoyancy and propagate between inner and outer turning points determined by the locations at which $\omega_{\alpha} \simeq N(r)$, where $N$ is the Brunt-Väisälä buoyancy frequency (Aerts et al. 2010). The inner turning point is very close to the stellar center $\left(r_{\alpha, \text { inner }} / R \simeq 10^{-3}\left(P_{\alpha} / \text { day }\right)^{-1}\right)$ and the outer turning point is near the radiative-convective interface at $\simeq 0.7 R$. Individual modes are described by the quantum numbers $(l, m, n)$, where $l$ is the spherical degree, $m$ is the azimuthal order, and $n$ is the radial order. Since the $g$-modes we consider are all very high order $(n \gtrsim 50)$, their properties are well approximated by the asymptotic WKB expressions given in WAQB. Using a $5 \mathrm{Gyr}$ old solar model from the EZ code (Paxton 2004), we find

$$
\omega_{\alpha} \simeq 7 \frac{l_{\alpha}}{n_{\alpha}} \omega_{0}
$$




$$
\gamma_{\alpha} \simeq 2 \times 10^{-11} \Lambda_{\alpha}^{2}\left(\frac{\omega_{0}}{\omega_{\alpha}}\right)^{2} \omega_{0}
$$

where $\Lambda_{\alpha}^{2}=l_{\alpha}\left(l_{\alpha}+1\right)$. The dominant linear damping mechanism of the high-order $g$-modes is radiative diffusion of the temperature fluctuations that accompany the mode density perturbations (Goodman \& Dickson 1998; Terquem et al. 1998). Shorter wavelength modes therefore have larger damping rates.

By plugging Equation (3) into Equation (7a), we can express the linear driving coefficient $U_{\alpha}$ in terms of the dimensionless linear overlap integral

$$
\begin{aligned}
I_{\alpha} & =\frac{1}{M R^{2}} \int d^{3} x \rho \boldsymbol{\xi}_{\alpha}^{*} \cdot \nabla\left(r^{2} Y_{2 m}\right) \\
& \simeq 2.5 \times 10^{-3}\left(\frac{\omega_{\alpha}}{\omega_{0}}\right)^{11 / 6},
\end{aligned}
$$

where the numerical expression assumes $l_{\alpha}=2, m_{\alpha}=m$ and is accurate for mode periods $P_{\alpha} \gtrsim 0.3$ day (Figure 11 of WAQB). Low-order, $l=2 g$-modes have large $I_{\alpha}$ but $\omega_{\alpha} \gg \Omega$; they comprise the quasi-static response of the fluid (the equilibrium tide). High-order, $l=2 g$-modes have small $I_{\alpha}$ but can nonetheless have large linear amplitudes if $\omega_{\alpha} \simeq 2 \Omega$; they comprise the resonant response of the fluid (the dynamical tide).

The three-mode coupling coefficient $\kappa_{\alpha \beta \gamma}$ is symmetric under the interchange of mode indices. Angular momentum conservation leads to the following angular selection rules for the three modes: (i) $l_{\alpha}+l_{\beta}+l_{\gamma}$ must be even, (ii) $m_{\alpha}+m_{\beta}+m_{\gamma}=0$, and (iii) the triangle inequality, $\left|l_{\alpha}-l_{\beta}\right| \leqslant l_{\gamma} \leqslant l_{\alpha}+l_{\beta}$. We focus on the parametric instability involving three-mode interactions between a high-order "parent" $g$-mode and a pair of high-order "daughter" $g$-modes whose summed frequency nearly equals the parent's frequency. For such a triplet, the coupling is strongest in the stellar core where the Lagrangian displacements of the modes peak. For a solar-type star (Appendix A in WAQB)

$$
\kappa_{\alpha \beta \gamma} \simeq 2 \times 10^{3}\left(\frac{T}{0.2}\right)\left(\frac{P_{\alpha}}{1 \text { day }}\right)^{2},
$$

where $P_{\alpha}$ is the period of the parent mode and $T \approx 0.1-1$ is an angular integral that depends on each mode's $l$ and $m$. The coupling occurs mostly near the parent's inner turning point $r_{\alpha, \text { inner }}$ and scales as $P_{\alpha}^{2}$ because the parent's displacement there varies as $\xi_{\alpha} \sim r_{\alpha, \text { inner }}^{-2} \sim P_{\alpha}^{2}$.

Although the equilibrium tide amplitude is large, its threemode coupling cancels significantly with nonlinear tidal driving $U_{\alpha \beta}$ (WAQB; see also Venumadhav et al. 2014). As a result, for a hot Jupiter system, the nonlinear dynamics are dominated by three-mode coupling to the dynamical tide; the energy dissipated in the equilibrium tide is small by comparison. ${ }^{1}$ We therefore restrict our mode networks to parent modes that comprise the dynamical tide response of the star (i.e., linearly resonant parents) and ignore the equilibrium tide response and nonlinear tidal driving.

\footnotetext{
1 Turbulent dissipation of the equilibrium tide within the convection zone yields $Q_{*}^{\prime} \sim 10^{8}-10^{9}$ (Penev \& Sasselov 2011), which is much larger than the $Q_{*}^{\prime}$ we find due to nonlinear damping of the dynamical tide (Section 5).
}

Finally, we assume that only linearly resonant modes (parents) have non-zero linear tidal forcing $U_{\alpha}$. This is justified because the linear forcing coefficient $U_{\alpha}$ is much smaller for daughter modes and their driving is far off resonance. Its secular effect will therefore be negligible compared to the resonant three-mode interactions. Ignoring such forcing allows us to adopt a convenient change of coordinates that significantly speeds up the integration of the amplitude equations (see Section 3.3).

\subsection{Instantaneous Orbital Decay Timescale}

The energy of the stellar modes is $E_{*} / E_{0}=\sum q_{\alpha} q_{\alpha}^{*}+$ $(1 / 3) \sum k_{\alpha \beta \gamma}\left(q_{\alpha} q_{\beta} q_{\gamma}+c . c\right)$. The rate of energy loss within a solitary star is therefore

$$
\begin{aligned}
\frac{\dot{E}_{*}}{E_{0}} & =\sum_{\alpha}\left(\dot{q}_{\alpha} q_{\alpha}^{*}+c . c\right)+\sum_{\alpha \beta \gamma} k_{\alpha \beta \gamma}\left(\dot{q}_{\alpha} q_{\beta} q_{\gamma}+c . c\right) \\
& =-2 \sum_{\alpha} \gamma_{\alpha} q_{\alpha} q_{\alpha}^{*}+\sum_{\alpha \beta \gamma} k_{\alpha \beta \gamma} \gamma_{\alpha}\left(q_{\alpha} q_{\beta} q_{\gamma}+c . c\right), \\
\dot{E}_{*} & \approx-2 \sum_{\alpha} \gamma_{\alpha} E_{\alpha},
\end{aligned}
$$

where we substituted the equations of motion (Equation (6)) for the mode amplitudes and neglected the terms from the three-mode couplings because they are much smaller than $E_{\alpha}=q_{\alpha} q_{\alpha}^{*} E_{0}$. This is the rate at which energy is dissipated within the star by radiative diffusion.

The dissipation of tidally excited stellar modes removes energy from the orbit, and the orbit therefore decays. We assume that the only dissipation in the system is due to the linear damping of waves excited within the star. Although the rotational energy of a synchronized planet increases as the orbit decays, this change is small compared to the corresponding change in orbital energy. Similarly, the energy in the excited stellar modes themselves may change with orbital period, but this also is a small effect (see Appendix A).

Because $\left|\dot{E}_{*} / E_{\text {orb }}\right| \ll \Omega$, where $E_{\text {orb }}=-G M M_{p} / 2 a$ is the orbital energy, we model the back-reaction on the orbit as a steady decrease in $E_{\text {orb }}$ of quasi-Keplerian circular orbits. The timescale of the instantaneous, orbital energy decay is then given by

$$
\tau_{E}=\frac{E_{\mathrm{orb}}}{\dot{E}_{*}}
$$

at each $P$, and we can compute a corresponding time-averaged decay timescale

$$
\left\langle\tau_{E}\right\rangle=\frac{a}{|\dot{a}|}=\frac{E_{\mathrm{orb}}}{\left\langle\dot{E}_{*}\right\rangle}
$$

where $\left\langle\dot{E}_{*}\right\rangle$ is the time-averaged energy dissipation rate with the average spanning several resonance peaks (if $\left\langle\tau_{E}\right\rangle \propto a^{n}$ then the "inspiral time" into the star will be $\left.\left\langle\tau_{E}\right\rangle / n\right)$. We describe our method of time-averaging in Appendix A. Using the language of linear tidal theory, $\left\langle\tau_{E}\right\rangle$ is often parameterized in terms of the star's tidal quality factor (Goldreich \& Soter 1966; see also Jackson et al. 2008)

$$
Q_{*}^{\prime}=7.5 \times 10^{6}\left(\frac{\left\langle\tau_{E}\right\rangle}{\mathrm{Gyr}}\right)\left(\frac{M_{p}}{M_{\mathrm{J}}}\right)\left(\frac{P}{\text { day }}\right)^{-13 / 3}
$$


where the expression assumes a circular orbit about a solar-type star and ignores dissipation within the planet. Although $Q_{*}^{\prime}$ is often taken to be a constant and fundamental property of the body, in general it depends on the companion mass, orbital frequency, and the tidal harmonics $(l, m)$.

\section{BUILDING AND INTEGRATING THE MODE NETWORKS}

In the absence of nonlinear three-mode interactions, the energy of a linearly driven parent mode $\alpha$ is (Equation (29) in WAQB)

$$
\frac{E_{\text {lin }}}{E_{0}}=\left|q_{\alpha, \text { lin }}\right|^{2}=\frac{\omega_{\alpha}^{2} U_{\alpha}^{2}}{\Delta_{\alpha}^{2}+\gamma_{\alpha}^{2}},
$$

where $\Delta_{\alpha}=\omega_{\alpha}-m_{\alpha} \Omega$ is the linear detuning. The parent is unstable to nonlinear three-mode interactions if there exists a pair of daughter modes $\beta$ and $\gamma$ such that $E_{\mathrm{lin}} \gtrsim E_{\mathrm{thr}}$, where the threshold energy is (see Appendix B)

$$
\frac{E_{\mathrm{thr}}}{E_{0}}=\frac{1}{4 \kappa_{\alpha \beta \gamma}^{2}}\left(\frac{\gamma_{b} \gamma_{\gamma}}{\omega_{\beta} \omega_{\gamma}}\right)\left[1+\left(\frac{\Delta_{\beta \gamma}}{\gamma_{\beta}+\gamma_{\gamma}}\right)^{2}\right] .
$$

Here $\Delta_{\beta \gamma}=\omega_{\beta}+\omega_{\gamma}+m_{\alpha} \Omega$ is the nonlinear detuning of the daughter pair. Daughter pairs with smaller $\left|\Delta_{\beta \gamma}\right|$ and larger $\left|\kappa_{\alpha \beta \gamma}\right|$ (i.e., stronger nonlinear coupling) yield smaller $E_{\mathrm{thr}}$ and are more readily unstable. In a three-mode system, unstable daughters with small initial amplitude undergo a phase of exponential growth at a rate

$$
\Gamma_{3 \mathrm{md}} \approx 2 \Omega\left|\kappa_{\alpha \beta \gamma}\right| \sqrt{E_{\mathrm{lin}} / E_{0}} .
$$

Eventually, the daughters reach an energy comparable to or greater than the parent's and the system reaches a nonlinear equilibrium (see Section 4.2 and Appendix B).

For the tide raised by even a $0.1 M_{\mathrm{J}}$ companion in a 3 day orbit, there are $\sim 10^{3}$ daughter pairs for which $E_{\mathrm{thr}}<E_{\text {lin }}$ (see Section 3.1). In Section 4, we systematically explore the dynamics of large multi-mode, multi-generation systems. In brief, we find that the parent drives many of the unstable daughters to large amplitudes and these daughters, in turn, drive granddaughters to large amplitudes, and so on. The total number of potentially unstable modes and the number of couplings is larger than the number we can integrate on a computer in a reasonable time $\left(\sim 10^{4}\right.$ and $\sim 10^{5}$, respectively). The issue then is whether we can reliably calculate the total tidal dissipation rate with a mode network that contains only a subset of the potentially unstable modes. We will present evidence in Section 4 that this is possible but we must build our networks carefully and systematically.

In Sections 3.1 and 3.2, we describe how we build networks consisting of sets of three-mode couplings (i.e., sets of triplets) and collective couplings, respectively. And in Section 3.3, we describe our method for integrating the coupled mode amplitude equation (Equation (6)).

\subsection{Building Three-mode Networks}

Although there are many daughter pairs with $E_{\mathrm{thr}}<E_{\mathrm{lin}}$, we show in Section 4 that pairs with low $E_{\text {thr }}$ dominate the dynamics of large multi-mode systems. We find that if we gradually increase the size of our networks by adding pairs

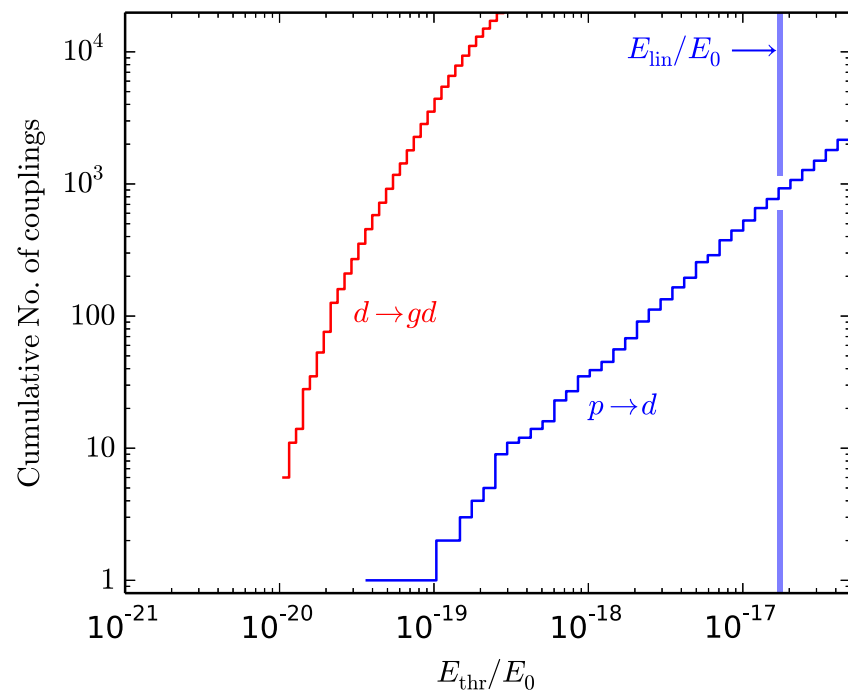

Figure 1. Cumulative distribution of $E_{\mathrm{thr}}$ for $M_{p}=0.1 M_{\mathrm{J}}$ and $P \simeq 3$ days (257928 s). (blue) Couplings from parents to daughters. The vertical blue line corresponds to the parent's linear energy $E_{\text {lin }}$. (red) Couplings from daughters to granddaughters.

with progressively higher $E_{\text {thr }}$, the system converges to a dissipation rate $\dot{E}_{*}$ that does not change significantly as we add even more modes. We must also include a sufficient number of generations (at least parents, daughters, and granddaughters) in order to obtain convergent results. Therefore, to build our mode networks, we comprehensively search the mode parameter space and construct, for each generation, a complete list of pairs ranked by $E_{\mathrm{thr}}$.

In order to carry out our search, we use the expressions for $\omega$, $\gamma$, and $\kappa$ (Equations (8a), (8b), and (10)) to solve for $E_{\mathrm{thr}}$. For a given parent mode $\alpha$, we first find the local minima of $E_{\mathrm{thr}}$ in the daughter parameter space $\left\{\left(n_{\beta}, l_{\beta}, m_{\beta}\right),\left(n_{\gamma}, l_{\gamma}, m_{\gamma}\right)\right\}$. In general, $E_{\mathrm{thr}}$ is minimized approximately where the sum in quadrature of $\Delta_{\beta \gamma}$ and $\gamma_{\beta}+\gamma_{\gamma}$ is minimized (modulo the angular selection rules and a relatively weak dependence on the angular integral $T$ ). Daughters with higher $l$ have smaller $\Delta_{\beta \gamma}$ (because they are more densely spaced in frequency) but larger $\gamma$ (because $\gamma \sim l^{2}$ ); the regions of small $E_{\text {thr }}$ therefore occur where these two countering effects are balanced. After finding the local minima, we expand our search around those minima and find pairs with progressively higher $E_{\mathrm{thr}}$. Because $\gamma \sim l^{2}$, at high enough $l$ the damping dominates detuning, and $E_{\text {thr }}$ increases with increasing $l$. We truncate our search upon reaching an $l_{\max }$ such that $E_{\mathrm{thr}}>E_{\mathrm{lin}}$ (i.e., a stable triplet). In practice, we find that for parent-daughter coupling, the dissipation $\dot{E}_{*}$ is dominated by the 10-100 lowest $E_{\mathrm{thr}}$ triplets.

Figure 1 shows the distribution of $E_{\text {thr }}$ for parent-daughter coupling assuming a $0.1 M_{\mathrm{J}}$ companion in an orbit near three days. There are a few pairs with very low $E_{\mathrm{thr}}$ because they just happen to have particularly small $\Delta_{\beta \gamma}$ despite having $l \lesssim 3$. There is a much larger sample of $\sim 10^{3}$ pairs that have larger $\Delta_{\beta \gamma}$ and/or $\gamma$ which still yield $E_{\text {thr }} \ll E_{\text {lin }}$.

We carry out a similar search when we consider the coupling of daughters to granddaughters. We show the distribution of $E_{\text {thr }}$ for daughter-granddaughter coupling in Figure 1 . The $E_{\text {thr }}$ of the most unstable daughter-granddaughter triplets is much smaller than the $E_{\mathrm{thr}}$ of the most unstable parent-daughter triplets (i.e., the red curve in Figure 1 is far to the left of the 
blue curve). Because $\kappa_{\alpha \beta \gamma} \propto \omega_{\alpha}^{-2}$ and $\Delta_{\beta \gamma} / \omega_{\alpha} \propto \omega_{\alpha}$, for low $E_{\text {thr }}$ pairs we find $E_{\text {thr }} \propto\left(\Delta_{\beta \gamma} / \kappa_{\alpha \beta \gamma} \omega_{\alpha}\right)^{2} \propto \omega_{\alpha}^{6}$. The factor of two decrease in frequency with each generation therefore means that $E_{\mathrm{thr}}$ decreases by $\sim 2^{6}$ (for a full discussion, see Appendix F). Physically, $E_{\text {thr }}$ decreases because lower frequency modes (i) penetrate deeper into the core where Lagrangian displacements are larger, and (ii) are more densely spaced in frequency and therefore can have smaller detunings. As a result, each generation is evermore susceptible than its predecessor to three-mode instabilities. This has important implications for the dynamics of large multi-mode, multigeneration systems, as we describe in Section 4.

\subsection{Building Collective Networks}

For high-order $g$-modes, the frequency spacing between neighboring modes is $|\Delta \omega| \sim \omega / n \ll \omega$. Therefore, if a pair of daughters is resonantly excited by a parent, there is a good chance that neighboring modes will also be resonantly excited by that same parent. The dynamics of such a system can be very different from that of a simple three-mode system; in particular, the daughters can grow as a single, collective unit with growth rates that are much higher than the three-mode case (see WAQB and Appendix E).

To appreciate why collective sets can grow so quickly, consider a simplified system in which a single parent mode $\alpha$ is coupled to $N$ daughter modes that are closely spaced neighbors in $(l, n)$ space. A study of the dynamics of such a system reveals that the modes all oscillate nearly in phase with each other. The equations of motion for each of the $N$ daughter modes can thus be approximated as

$$
\begin{aligned}
\dot{q}_{\beta}+\left(i \omega_{\beta}+\gamma_{\beta}\right) q_{\beta} & =i \omega_{\beta} \sum_{\gamma} \kappa_{\alpha \beta \gamma}^{*} q_{\alpha}^{*} q_{\gamma}^{*} \\
& \simeq i \omega_{\beta} N \kappa_{\alpha \beta \gamma}^{*} q_{\alpha}^{*} q_{\gamma}^{*} .
\end{aligned}
$$

The dynamics look like the three-mode case, but with an effective coupling coefficient that is $N$ times larger. In particular, the instability growth rates (threshold amplitudes) are approximately $N$ times larger (smaller) than the threemode case.

When building our mode networks, we use separate algorithms to search for collective sets and three-mode sets. A simple but incomplete way to build collective sets is to first find a daughter with a frequency nearly equal to half that of the parents and then progressively add neighbors with $\Delta n= \pm 1$, $\pm 2, \pm 3, \ldots$. At first, $E_{\text {thr }}$ will decrease as more modes are added and $N$ increases. However, for large enough $\Delta n$, the detuning of the outer most modes becomes so large that adding more modes does not decrease $E_{\mathrm{thr}}$ any further. ${ }^{2}$ More detail is provided in Appendix E.4. Although this method naturally picks out collective sets (and is similar to the approach described in WAQB), it potentially misses many collectively unstable modes. For example, there can be distinct groups of modes that are not nearby neighbors and yet together form a collective set. For this reason, we use a more sophisticated method when building collective networks. We describe this method in Appendix D.

\footnotetext{
2 In addition, the magnitude of $\kappa_{\alpha \beta \gamma}$ becomes small for $\Delta n \gtrsim n_{\alpha}$ because the coupled daughters are no longer spatially resonant with the parent (see Figure 12 in WAQB).
}

In Section 4, we show that collective sets are excited and initially grow much more rapidly than three-mode sets. However, when the entire network ultimately reaches its nonlinear equilibrium and saturates, we find that the collective sets do not alter $\dot{E}_{*}$ significantly. We therefore find that we can accurately calculate $\dot{E}_{*}$ with networks that include only threemode sets.

\subsection{Integration Method}

We integrate the amplitude equation (Equation (6)) for each mode of a network using an adaptive step-size 4th-5th order Runge-Kutta integrator. Our integrations take advantage of a convenient change of coordinates, also described in Brink et al. (2005). The integration step size is limited by the fastest frequency in the equations. Because the linear and nonlinear forcings all involve resonant interactions ${ }^{3}$, the linear and nonlinear detunings $\left(\Delta_{\alpha}\right.$ and $\left.\Delta_{\beta \gamma}\right)$ are all small $(\ll \Omega)$. In fact, the fastest timescale in these equations is typically the natural frequency $\omega_{\alpha}$ of each mode. By changing coordinates to $x_{\alpha}=q_{\alpha} e^{i \omega_{\alpha} t}$, we can remove these frequencies from the equations of motion at the cost of adding a slowly varying time-dependent term to each three-mode coupling. This increases the typical integration step size by approximately the ratio of $\omega_{\alpha}$ to the detuning $\left(\approx 10^{2}-10^{4}\right)$.

In order to further speed up the integrations, we parallelize across multiple CPUs. We achieve this using standard parallelization techniques, with care taken to equally distribute the amount of work across each CPU. For example, the computation of $\dot{x}_{\alpha}$ scales with the number of couplings included for that mode. Therefore, when we parallelize the computation of $\dot{x}_{\alpha}$ by splitting modes among processes, we attempt to divide modes into sets with equal numbers of couplings, rather than equal numbers of modes. We test several different parallelization methods, including an implementation using Python's subprocess module, Python's multiprocessing module, and a Python wrapper for OpenMPI. All our implementations scale better than $N_{\text {CPU }}^{-0.8}$, although which implementation is fastest depends on specifics of the hardware. The Python multiprocessing implementation generally performed best, and parallelized across $152.7 \mathrm{GHz}$ Quad-Core AMD Opteron Processors, it takes $\simeq 40 \mathrm{~s}$ to integrate one of our largest networks $\left(\simeq 2.4 \times 10^{4}\right.$ modes with $\simeq 3 \times 10^{5}$ couplings) through 10 orbital periods.

\section{MODE DYNAMICS}

In order to build up intuition for the results from large multimode, multi-generation networks, we describe the mode dynamics of increasingly complicated networks. We begin in Section 4.1 with a network that consists only of linearly resonant parents (i.e., we ignore all nonlinear couplings) and show that our simulations recover the dissipation rates of standard linear theory. In Section 4.2 we couple linearly resonant parents to unstable daughter modes but do not allow the daughters to couple to granddaughters. We find that including even just this first generation of nonlinear couplings enhances the dissipation rate by a factor of $\approx 100(\approx 10)$ relative to the linear result for $P=1$ day (3 day) and $M_{p}=M_{\mathrm{J}}$. In Section 4.3 we allow the daughters to couple to granddaughters and find that this further enhances the dissipation, yielding a

\footnotetext{
3 For reasons described in Section 2.2, we assume that only the linearly resonant parent modes have a non-zero linear tidal forcing $U_{\alpha}$.
} 

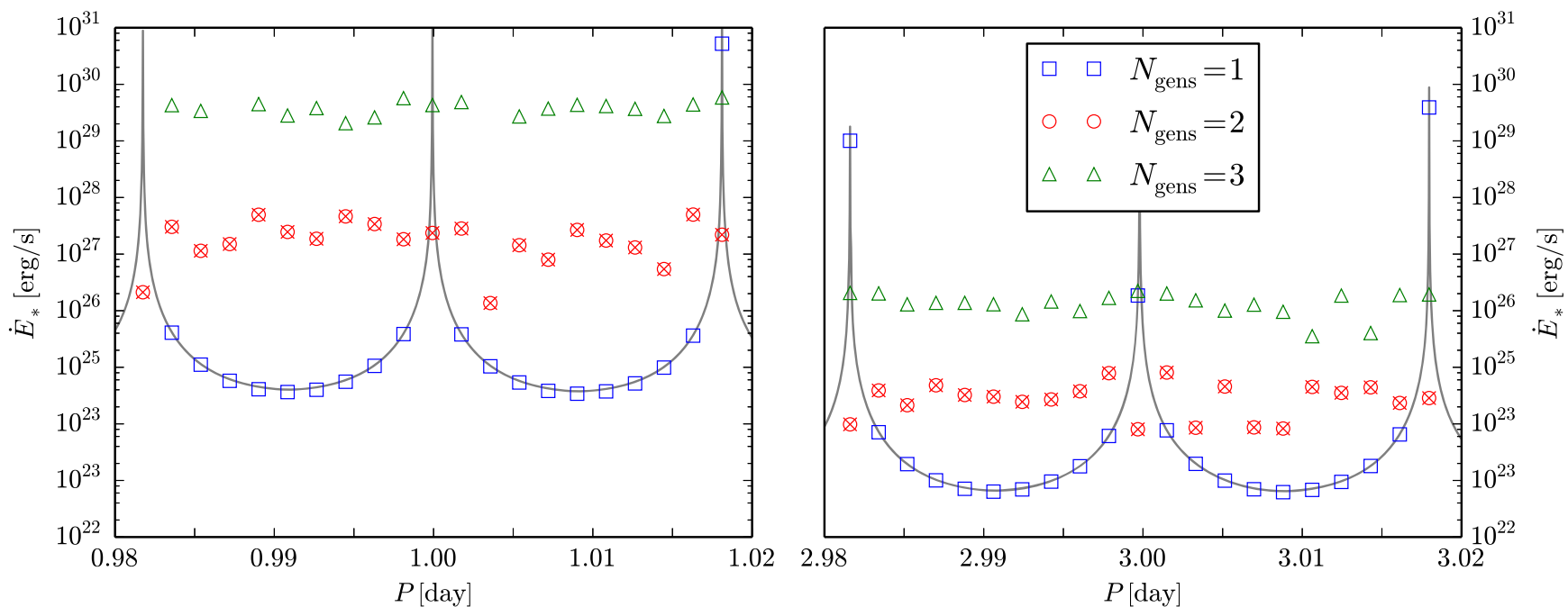

Figure 2. Energy dissipation rate $\dot{E}_{*}$ as a function of orbital period $P$ for networks that include different numbers of mode generations $N_{\text {gens }}$. We show results for $M_{p}=M_{\mathrm{J}}$ and orbits near $P=1$ day (left panel) and $P=3$ days (right panel). The sharp linear resonance peaks occur when the tidal driving frequency is resonant with an $l=2 g$-mode of the star. (blue squares) Networks with only the ten most resonant parents. (red circles) Networks with parents and daughters. (green triangles) Networks with parents, daughters and granddaughters (shown here are the results of our "reference network"; see Section 4.6). (gray lines) The analytic estimate of the steady state dissipation for parent-only networks that contain the 100 most resonant parents. (red crosses) The analytic estimate of the steady state dissipation for parent-daughter networks (see Equation (21)). We do not have an analytic estimate for parent-daughter-granddaughter networks.
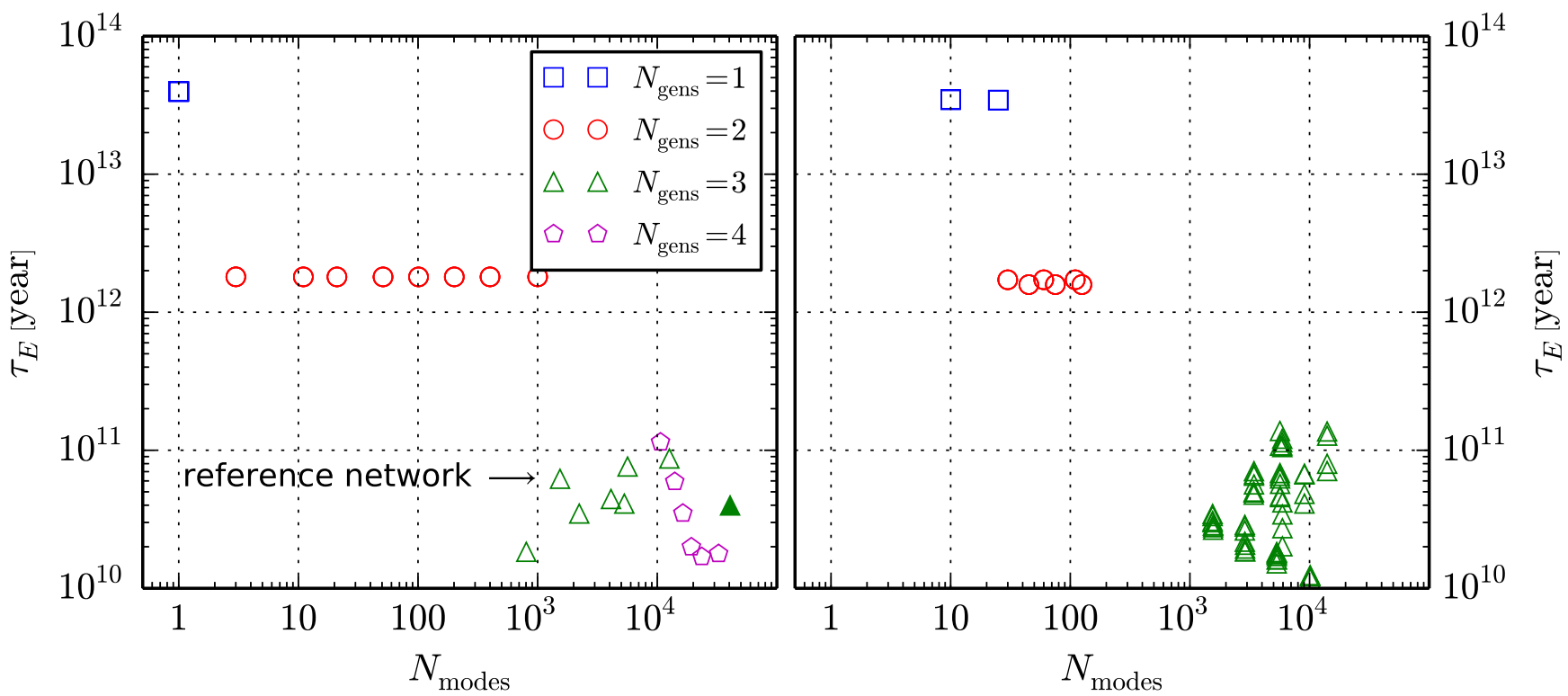

Figure 3. Orbital decay timescale $\tau_{E}$ as a function of the number of modes $N_{\text {modes }}$ for networks that include different numbers of mode generations $N_{\text {gens }}$. We show results for a Jupiter-mass companion orbiting at a period near three days (257928 s, chosen to be approximately half way between a resonance trough and resonance peak for our stellar model). The left panel networks have a single parent mode and the right panel networks have 10 or 25 parent modes. (blue squares) Networks with only parents. (red circles) Networks with parents and daughters. (green triangles) Networks with parents, daughters, and granddaughters. The filled triangle corresponds to a reference network with collective granddaughter modes added. (purple pentagons) Networks with parents, daughter, granddaughters, and greatgranddaughters. The structure of the networks with only a single parent (left panel) are as follows: $N_{\text {gens }}=2$ networks range from one daughter pair up to 500 daughter pairs. $N_{\text {gens }}=3$ networks mostly correspond to 10 daughter pairs and either 25,50 (our reference network; Section 4.6), 75, 150, or 200 granddaughter pairs per daughter, although there are networks with 50 and 200 daughter pairs, each with 50 granddaughter pairs per daughter. $N_{\text {gens }}=4$ networks are all extensions of our reference network, adding 10,25, 50,100, 200, or 500 great-granddaughter pairs per granddaughter. The structure of the multi-parent networks (right panel) are as follows: either 10 or 25 parent modes; 0, 10, 25, or 50 daughter pairs per parent; and either 0, 50, 100, or 200 granddaughter pairs per daughter.

rate that is $\approx 10^{5}\left(\approx 10^{3}\right)$ times larger than the linear result for $P=1$ day (3 day) and $M_{p}=M_{\mathrm{J}}$. We find in Sections 4.4 and 4.5 that the dissipation rates do not change significantly when we include even more generations (great granddaughters and beyond) and collective sets, respectively, suggesting that the system has reached a convergent, saturated state. In Section 4.6 we explore the minimum network size needed to attain such a convergent state.

\subsection{Linear Parents Only}

If we include only linearly driven parents in the network, then $\left|\dot{E}_{*}\right|=2 \sum \gamma_{\alpha}\left(E_{\alpha}\right)_{\text {lin }}$, where the parent linear energy $\left(E_{\alpha}\right)_{\text {lin }}$ is given by Equation (15). The dissipation is typically dominated by the most linearly resonant parent, although other modes can contribute if no single mode is particularly resonant. In Figure 2, we show $\dot{E}_{*}$ due to the ten most resonant parents over a small range in orbital period. In the absence of nonlinear 
interactions, the orbit evolves rapidly through the sharp resonance peaks where $\dot{E}_{*}$ is large. As we describe in Appendix A, the time average dissipation rate is the sum of the instantaneous $\dot{E}_{*}$ weighted by the amount of time spent at that period (see also Goodman \& Dickson 1998). In the left panel of Figure 3, we show $\tau_{E}$ (Equation (12)) due to the single most resonant parent assuming $P \simeq 3$ day, $M_{p}=M_{\mathrm{J}}$. An analytic calculation using Equations (8b) and (15), and assuming $\Delta_{\alpha} \sim \omega_{\alpha} / 2 n_{\alpha} \gg \gamma_{\alpha}$, yields (see Appendix G)

$$
\left\langle\tau_{E}\right\rangle_{\text {lin }} \simeq 1.4 \times 10^{12}\left(\frac{M_{p}}{M_{\mathrm{J}}}\right)^{-1}\left(\frac{P}{\text { day }}\right)^{3} \text { year }
$$

which translates to

$$
Q_{*, \text { lin }}^{\prime} \simeq 1.1 \times 10^{10}\left(\frac{P}{\text { day }}\right)^{-4 / 3} .
$$

This is in good agreement with our numerical integrations. The $M_{p}$ dependence of $\left\langle\tau_{E}\right\rangle_{\text {lin }}$ is due to the linear forcing coefficient $U_{\alpha}$ and the dependence on $P$ is due to a combination of $\gamma_{\alpha}, \Delta_{\alpha}$, and $U_{\alpha}$. We will show that when we include nonlinear interactions, the instantaneous decay time has a dramatically different magnitude and scaling with $M_{p}$ and $P$.

The right panel of Figure 3 shows the results for the same parameters as the left panel, but for networks with either the 10 or 25 most resonant parents. Despite including these additional modes, the instantaneous decay time is nearly identical in both cases. More generally, we find that including multiple parents has very little effect on the total dissipation (even for networks with nonlinear interactions) as long as $P \lesssim 4$ days. This is because, for $P \lesssim 4$ days, the parent mode spacing is sufficiently sparse that the most resonant parent typically has a much larger $E_{\text {lin }}$ than the neighboring parents, and it therefore dominates the dynamics (Appendix C).

\subsection{Parents and Daughters}

In Figure 4 we show the mode dynamics of networks that include daughters (but not granddaughters) coupled to a linearly resonant parent. The top panel shows a simple threemode system involving a parent coupled to only its lowest $E_{\text {thr }}$ daughter pair. Initially, the daughters are at small energy and the parent is at its linear energy $E_{\text {lin }}$. Because $E_{\text {lin }}>E_{\mathrm{thr}}$, the system is unstable and the daughters undergo a rapid initial growth at the rate given by Equation (17). Eventually the system reaches a nonlinear equilibrium in which the parent has energy $E_{\alpha}=E_{\mathrm{thr}}$ and the daughters have energy $E_{\beta, \gamma} \simeq\left|U_{\alpha} / 2 \kappa_{\alpha \beta \gamma}\right| E_{0}$ (see Appendix B and WAQB).

The middle panel of Figure 4 shows the same parent now coupled to the ten lowest $E_{\text {thr }}$ daughter pairs. Because $E_{\text {lin }}>E_{\text {thr }}$ for all ten pairs, initially all the daughters grow. However, eventually the parent energy drops to the minimum $E_{\text {thr }}$ and only the lowest threshold daughter pair remains excited; the other daughters decay due to linear damping. The nonlinear equilibrium of this system is therefore equivalent to the three-mode network shown in the top panel.

The network shown in the middle panel of Figure 4 assumes that each of the ten triplets only share a parent. If the triplets also share daughters (e.g., daughter $a$ couples to daughter $b$ and daughter $c$ ), then the dynamics can be more complicated. Such a network is shown in the bottom panel of Figure 4 and illustrates how such additional couplings parasitically excite

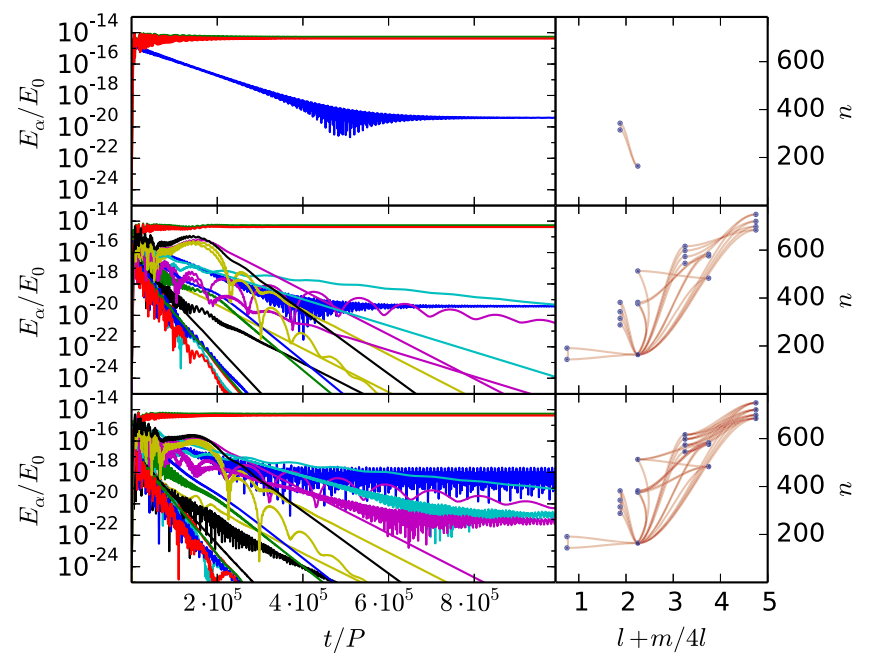

Figure 4. Parent-daughter networks $\left(N_{\text {gens }}=2\right)$ with different structures. The left panels show the energy $E_{\alpha}$ of each mode as a function of time. In each of these panels, the parent is the blue line and is initially at $E_{\text {lin }}$ but ultimately settles into a nonlinear equilibrium at an energy $E \ll E_{\text {lin }}$. The right panels show the coupling diagrams in the $n-l$ plane, with circles representing the included modes and line connections indicating the coupling structure. (top) A parent and the lowest $E_{\mathrm{thr}}$ daughter pair. (middle) A parent and the ten lowest $E_{\text {thr }}$ daughter pairs with each daughter mode couple to only one other daughter mode. (bottom) A parent and the ten lowest $E_{\text {thr }}$ pairs with all allowed couplings between daughters.

other daughters. ${ }^{4}$ O'Leary \& Burkart (2014) consider a similar mechanism in order to explain the odd resonances observed in the KOI-54 light curve. Despite these additionally excited modes, the lowest $E_{\mathrm{thr}}$ pair still dominates the dissipation.

In Figure 3, we show $\tau_{E}$ for networks that include only parent-daughter couplings $\left(N_{\text {gen }}=2\right)$ assuming $P \simeq 3$ day, $M_{p}=M_{\mathrm{J}}$. We find that at this period parent-daughter coupling decreases $\tau_{E}$ by a factor of $\sim 10$ relative to the linear result. Numerically, both $\tau_{E}$ and $\left\langle\tau_{E}\right\rangle$ are nearly independent of the number of daughter modes in the parent-daughter networks because the daughter pair with the lowest $E_{\mathrm{thr}}$ dominates the dynamics and dissipation. The other daughters, while excited, do not reach significant amplitudes and therefore have little effect. Even for large numbers of modes, parent-daughter systems behave much like those shown in the middle and bottom panels of Figure 4 . We therefore find that $\left\langle\tau_{E}\right\rangle$ is wellapproximated by the analytic calculation that assumes only one parent and its single lowest $E_{\text {thr }}$ daughter pair (see Appendix G and Figure 10)

$$
\left\langle\tau_{E}\right\rangle_{\mathrm{p}-\mathrm{d}} \simeq 2.0 \times 10^{11}\left(\frac{P}{\text { day }}\right)^{19 / 6} \text { year }
$$

assuming $l=1$ daughters and

$$
Q_{*, \mathrm{p}-\mathrm{d}}^{\prime} \simeq 1.5 \times 10^{9}\left(\frac{M_{p}}{M_{\mathrm{J}}}\right)\left(\frac{P}{\text { day }}\right)^{-7 / 6} .
$$

The agreement between the numerical result for parentdaughter networks containing multiple daughters and the above three-mode estimate is further illustrated in Figure 2. The open circles show the numerically computed $\dot{E}_{*}$ of a parent-daughter network consisting of the $\simeq 20$ lowest $E_{\text {thr }}$

\footnotetext{
$\overline{4}$ This is a form of nonlinear inhomogeneous driving and is described in WAQB.
} 
daughter modes. Each of these open circles is covered by an "x," which represent the analytically computed $\dot{E}_{*}$ assuming only the minimum $E_{\text {thr }}$ daughter pair.

We will now see, however, that the dynamics are much more complicated when granddaughters are included, with many more modes excited to significant amplitudes.

\subsection{Parents, Daughters, and Granddaughters}

In the absence of granddaughter couplings, the lowest $E_{\text {thr }}$ daughter pair settles into a nonlinear equilibrium at an energy $E_{\beta, \gamma} \simeq\left|U_{\alpha} / 2 \kappa_{\alpha \beta \gamma}\right| E_{0}$. However, as discussed in Section 3.1, there are many granddaughter pairs that are unstable to such high energy daughters (see Figure 1). The parent-daughter solutions of the previous section are therefore unstable and never realized.

For very small networks that include granddaughters we sometimes observe periodic limit cycles. However, for even slightly larger networks with more complicated coupling topologies, the limit cycles begin to take on a more chaotic appearance. And for the very large networks that we find yield convergent dissipation results $\left(\gtrsim 10^{3}\right.$ modes), the dynamics cease to display any clear limit cycle behavior over long timescales and instead show persistent large amplitude fluctuations involving many excited modes (Figure 7).

We can roughly understand the behavior of these networks using intuition from simple three-mode systems. Initially, an unstable, linearly driven parent excites daughters to large energy. The daughters drain energy from the parent and the parent's energy drops. However, the daughters then excite granddaughters and the daughters' energy drops. The daughters no longer drain enough energy from the parent and the parent begins to recover due to linear driving. The rising parent excites the daughters again and the cycle restarts.

Unstable granddaughters have lower frequencies and, in general, higher $l$ than the parents and daughters. They therefore often have much smaller radial wavelengths (i.e., much larger $n)$ and thus much larger linear damping rates $\left(\gamma \propto n^{2}\right)$. This means that granddaughters can dissipate energy more rapidly than daughters even if they are at a lower amplitude.

In Figure 3 we show $\tau_{E}$ for large networks that include parents, daughters, and granddaughters $\left(N_{\text {gen }}=3\right)$ assuming $P \simeq 3$ day and $M_{p}=M_{\mathrm{J}}$. We see that networks with granddaughters are more dissipative than parent-daughter only networks and yield $\tau_{E} \lesssim 10^{-3} \tau_{E \text {,lin }}$ at $P \simeq 3$ day. The figure also shows $\tau_{E}$ as a function of the number of modes $N_{\text {modes }}$ in the network. We find a systematic uncertainty in $\tau_{E}$ associated with the structure of the network. However, this uncertainty is small compared to the increase in dissipation associated with the inclusion of granddaughter modes. In this sense, we find that $\tau_{E}$ is not particularly sensitive to the number of granddaughters nor to the details of the network structure as long as the number of granddaughter modes is sufficiently large $\left(\gtrsim 10^{3}\right)$. We illustrate this point further when we discuss our reference networks in Section 4.6.

When we build networks with larger $N_{\text {modes }}$, we do so by adding modes of increasingly larger $E_{\mathrm{thr}}$ (see Section 3.1). The fact that $\tau_{E}$ does not change as we increase $N_{\text {modes }}$ above $\sim 10^{3}$ suggests that the lowest $E_{\text {thr }}$ modes dominate the energy dissipation and, therefore, that our method for building networks reliably captures the bulk of the dissipation. We illustrate this more explicitly in Figure 5, which shows that the overwhelming majority of the energy is dissipated by the

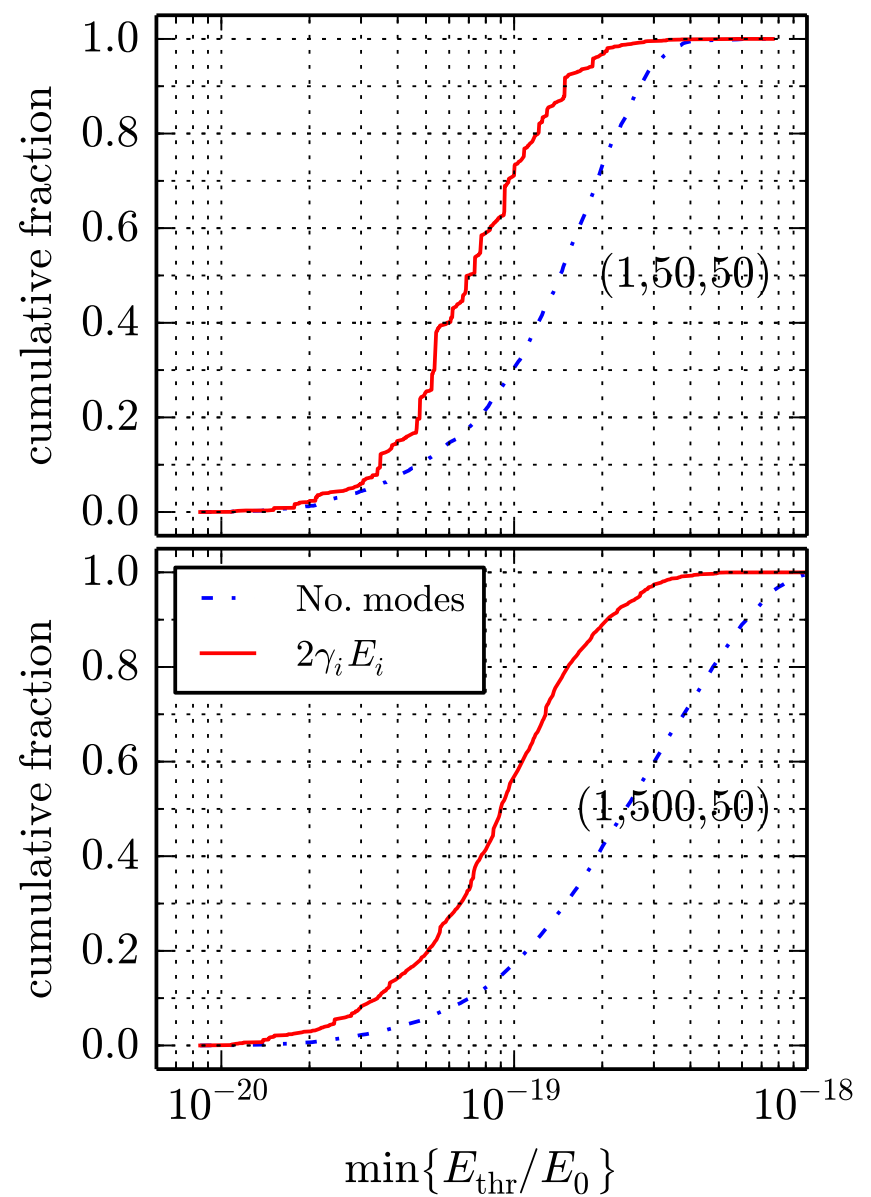

Figure 5. Cumulative distributions of the number of modes included in the network (blue) and the energy dissipation rate of the modes (red) as a function of each modes $E_{\mathrm{thr}}$. For granddaughter modes coupled to more than one daughter, we take their minimum $E_{\mathrm{thr}}$. (top) a network consisting of one parent, 50 daughter pairs, and 50 granddaughter pairs per daughter. (bottom) a network consisting of one parent, 500 daughter pairs, and 50 granddaughter pairs per daughter. Approximately $90 \%$ of the energy dissipation is due to the first $50 \%$ (40\%) of modes ordered by $E_{\mathrm{thr}}$ for the smaller (larger) network.

modes with the lowest $E_{\mathrm{thr}}$ and that modes with ever larger $E_{\mathrm{thr}}$ contribute less and less to the total dissipation. This suggests that selecting modes based on their $E_{\text {thr }}$ identifies the dynamically relevant couplings and that including enough modes in this way yields convergent results.

\subsection{Great Granddaughters and Beyond}

If daughters and granddaughters are excited, what about great granddaughters $\left(N_{\text {gen }}=4\right)$ and so on? This seems particularly likely given that $E_{\text {thr }} \propto \omega^{-6}$ (see Section 3.1). Indeed, based on our experiments with networks that include up to five generations, we observe that the cascade continues into many generations. However, as long as we include enough modes, we find that the total dissipation rate plateaus once we include granddaughters. In effect, we do not need to resolve the innermost scales of the energy cascade in order to obtain an accurate estimate of $\dot{E}_{*}$.

We illustrate this in the left panel of Figure 3, which shows $\tau_{E}$ for networks that go up to $N_{\mathrm{gen}}=4$. There are dramatic decreases in $\tau_{E}$ when going from just parents $\left(N_{\text {gen }}=1\right)$ to parents and daughters $\left(N_{\text {gen }}=2\right)$, and again when adding granddaughters $\left(N_{\text {gen }}=3\right)$. However, we see only a slight 


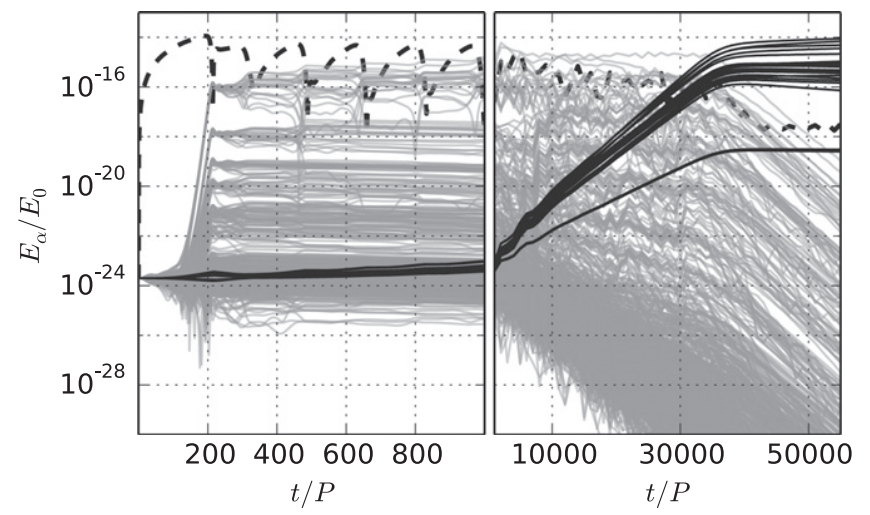

Figure 6. Parent-daughter networks $\left(N_{\text {gens }}=2\right)$ that include collective sets in addition to three-mode sets. (black dashed line) the parent mode. (black solid line) daughters selected with three-mode algorithm. (grey solid lines) daughters selected with collective algorithms. In the left panel we show the dynamics of the system at early times. Several separate sets of collective modes are excited, grow rapidly, and reach significant energies by $t \approx 200 P$. In the right panel we show the dynamics out to much longer times. The slowly growing set of unstable three-mode daughters eventually reach large amplitudes at $t \approx 3 \times 10^{4} P$. This drives the parent's amplitude down and the collective modes decay away. We only plot one out of every ten collective modes from this simulation.

decrease in $\tau_{E}$ when we add great-granddaughters $\left(N_{\text {gen }}=4\right)$. In particular, for sufficiently large $N_{\text {gen }}=4$ networks $\left(N_{\text {modes }} \gtrsim 2 \times 10^{4}\right)$, we find that $\tau_{E}$ plateaus at a value that is only $\simeq 3$ times smaller than that of our $N_{\text {gen }}=3$ reference network at this $P$. This suggests that truncating at $N_{\text {gen }}=3$ yields reasonably accurate estimates of $\tau_{E}$.

\subsection{Dynamics of Collective Networks}

WAQB showed that sets of modes can be collectively unstable even if each pair within the set is stable by itself. We describe algorithmic approaches to identify and select such sets of modes in Section 3.2 and Appendix E. These collective sets can have growth rates that are hundreds of times faster than the separate three-mode growth rates (Equation (17)). We illustrate this in the left panel of Figure 6, which shows several collectively unstable sets of modes being rapidly driven to large amplitudes after only a few hundred orbital periods. However, for $P \lesssim 10$ days (see Figure 7 of WAQB), the lowest $E_{\text {thr }}$ for individual three-mode triples is lower than the collective stability threshold. This means that after the collective modes grow rapidly, the parent's amplitude is still large enough to drive three-mode triples. The right panel of Figure 6 shows that the slowly growing three-mode triples eventually reach large amplitudes and drive the parent below the collective instability threshold. At that point, all the collective modes "turn off" and decay, leaving the steady state predicted by simple three-mode systems.

The network in Figure 6 only includes parents and daughters. However, because these daughters are unstable to granddaughter interactions (Section 4.3), the collective modes may not decay forever but instead may saturate at non-trivial amplitudes. In principal, because collective modes can have significantly larger $l$ than the minimum $E_{\mathrm{thr}}$ pair and thus larger damping coefficients, they may dissipate energy more rapidly. Nonetheless, numerical experiments reveal that sufficiently large networks constructed out of only three-mode pairs yield nearly the same $\dot{E}_{*}$ as networks that also include collective excitations. This can be seen in Figure 3; the filled triangle corresponds to the reference network (Section 4.6) with the addition of collective granddaughters. Because collective networks are expensive to simulate and do not change the calculated $\dot{E}_{*}$, from here on we do not include them in our calculations.

\subsection{Reference Network Integrations}

To summarize, we find that networks with parents, daughters, and granddaughters yield convergent dissipation results as long as they include a large enough number of low $E_{\text {thr }}$ daughter and granddaughter modes. Moreover, it is not necessary to include collective sets of daughters and granddaughters; although they can modify the dynamics somewhat, the low $E_{\text {thr }}$ three-mode sets ultimately model the total dissipation well.

Further numerical experiments reveal that a network consisting of one parent, its $\simeq 20$ lowest $E_{\text {thr }}$ daughters, and $\simeq 1500$ low $E_{\mathrm{thr}}$ granddaughters is sufficiently large that it yields convergent results while still allowing us to efficiently explore a range of $M_{p}$ and $P$ (see caption of Figure 3). We use this as our "reference network" when computing $\left\langle\tau_{E}\right\rangle$ as a function of $M_{p}$ and $P$ in Section 5. That such a network is sufficiently large can be gleaned from the left panel of Figure 3, which shows that the reference network $\tau_{E}$ is very similar to $N_{\text {gen }}=3$ or 4 networks with $N_{\text {modes }} \gtrsim 10^{4}$.

We demonstrate this further in Figure 7, which shows the mode energy and the effective number of modes participating in the dissipation $N_{\text {eff }}$ as a function of time for four different orbital periods. We estimate $N_{\text {eff }}$ by computing

$$
N_{\text {eff }}=\exp \left(-\sum_{\alpha} p_{\alpha} \ln p_{\alpha}\right),
$$

where

$$
p_{\alpha}=\frac{\gamma_{\alpha} q_{\alpha} q_{\alpha}^{*}}{\sum_{\alpha} \gamma_{\alpha} q_{\alpha} q_{\alpha}^{*}}
$$

This statistic is related to the Shannon entropy and is similar to one used in Brink et al. (2005). If all modes contribute equally to the dissipation, then $p_{\alpha}=1 / N_{\text {modes }}$ for each mode and $N_{\text {eff }}=N_{\text {modes }}$. We see in Figure 7 that the dynamics are complicated, with many excited modes. We also see that the mode energy and $N_{\text {eff }}$ increase at shorter $P$ and near linear resonances. However, $N_{\text {eff }} \ll N_{\text {modes }}$ while both the peak energy of the modes and $N_{\text {eff }}$ remain nearly constant after $\sim 100,000$ orbits, indicating that our reference networks are sufficiently large. The behavior of the networks as a whole is what is important here, rather than the dynamics of any individual mode. Individual modes fluctuate significantly but the overall dynamics do not change over long timescales.

We now describe our procedure for calculating $\left\langle\tau_{E}\right\rangle$ as a function of $M_{p}$ and $P$, the results of which we present in Section 5. For each reference network run at a given $\left(M_{p}, P\right)$ point, we simulate at least $5 \times 10^{5}$ orbits in order to allow transient effects from initial conditions to die away. ${ }^{5}$ Using Equation (11), we then compute the average $\dot{E}_{*}$ over the last $\sim 5 \times 10^{4}$ orbits of the integration. We do this in order to

\footnotetext{
5 This is true of all networks in Figure 3 as well, with the exception of the largest $N_{\text {gens }}=4$ network and the collective network, where we were computationally limited to shorter (but still convergent) integrations.
} 

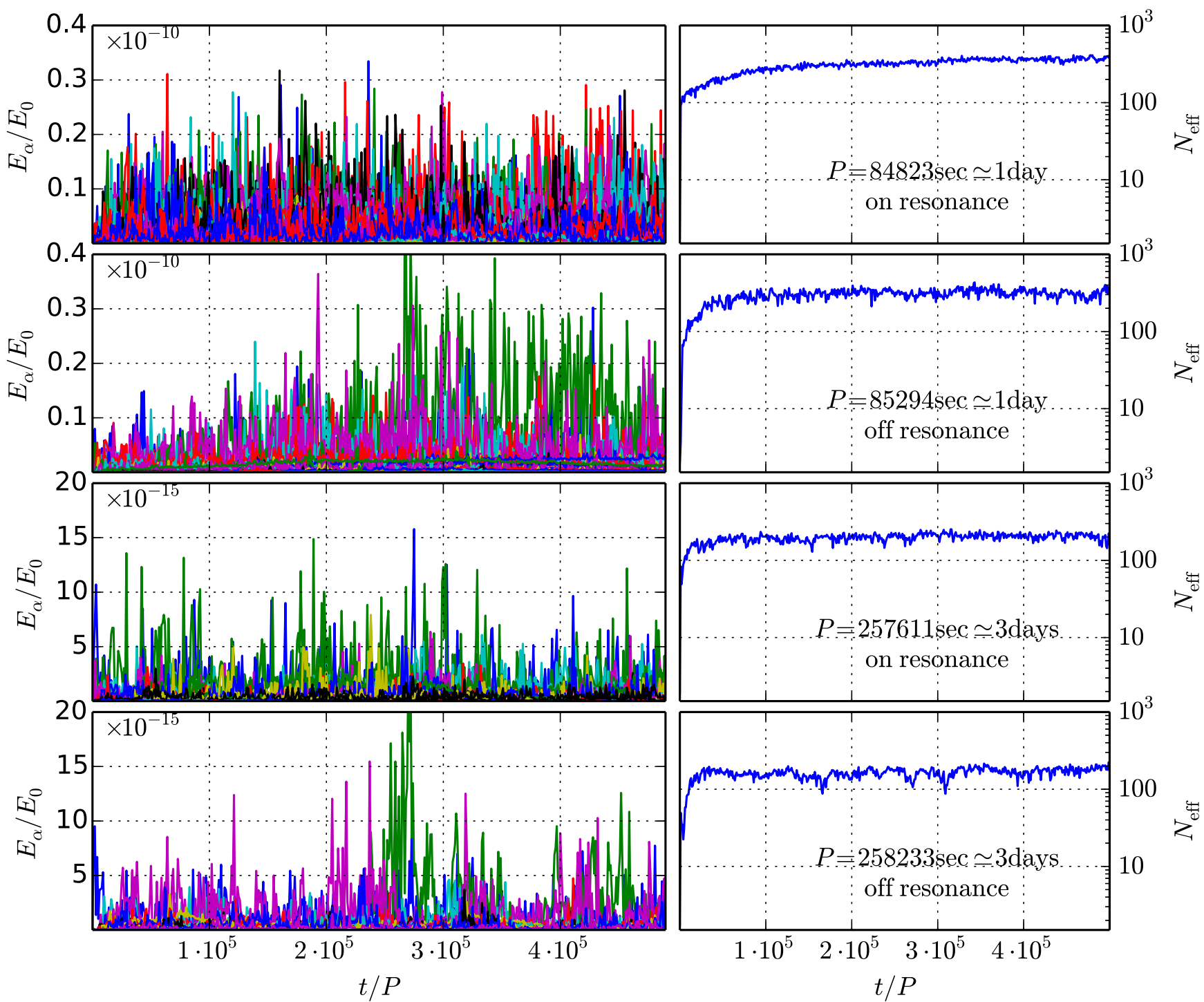

Figure 7. Mode energy $E_{\alpha}$ (left panels) and effective number of modes $N_{\text {eff }}$ (right panels) as a function of time for reference networks with $M_{p}=M_{\mathrm{J}}$. (top to bottom) $P \simeq 1$ day on resonance, off resonance, $P \simeq 3$ day on resonance, off resonance. For clarity, on the left we plot only one out of every 25 granddaughter modes included in the simulation.

average over the rapid fluctuations in dissipation that characterize the nonlinear equilibria, which correspond to rms variations at roughly a $10 \%$ level. We also find that $\dot{E}_{*}$ depends slightly (factor of $\approx 2$ ) on how close the parent is to a linear resonance peak (see the $N_{\text {gen }}=3$ results in Figure 2). In order to compute the average dissipation rate, we must therefore average the results over several resonance peaks. We do this by performing 21 separate integration runs, each at a slightly different orbital period $(\delta P \ll P)$ chosen such that the runs span three resonance peaks. We then calculate the average $\dot{E}_{*}$ weighted by the amount of time spent at that orbital period (Appendix A) and use this $\left\langle\dot{E}_{*}\right\rangle$ to estimate $\left\langle\tau_{E}\right\rangle$ via Equation (13).

\section{RESULTS}

In this section we present the results of integrating the coupled amplitude Equation (6) using the procedure and reference networks described in Section 4.6. Figures 8 and 9 show $\left\langle\tau_{E}\right\rangle$ as a function of $M_{p}$ and $P$. We find that $\left\langle\tau_{E}\right\rangle$ depends strongly on $P$ and only mildly on $M_{p}$. Our numerical results are well approximated by the fit

$$
\left\langle\tau_{E}\right\rangle=4.1 \times 10^{7}\left(\frac{M_{p}}{M_{\mathrm{J}}}\right)^{-0.5}\left(\frac{P}{\text { day }}\right)^{6.7} \text { year }
$$

over the range $0.5 \lesssim M_{p} / M_{\mathrm{J}} \leqslant 3$ and $P \lesssim 4$ days. This matches our numerical results to within a factor of $\simeq 2$ over this range, which is comparable to systematic modeling uncertainties due to differences in the network structure of large $N_{\text {gens }}=3,4$ networks (see Figure 3). By Equation (14), this corresponds to a stellar tidal quality factor

$$
Q_{*}^{\prime}=3.0 \times 10^{5}\left(\frac{M_{p}}{M_{\mathrm{J}}}\right)^{0.5}\left(\frac{P}{\text { day }}\right)^{2.4} .
$$

Thus, for $M_{p} \gtrsim 0.5 M_{\mathrm{J}}$ and $P \lesssim 2$ day we find that $\left\langle\tau_{E}\right\rangle$ is small compared to the main-sequence lifetime of a solar-type star. For $M_{p} \lesssim 0.5 M_{\mathrm{J}}$, we find that although $\left\langle\tau_{E}\right\rangle$ increases significantly, it can still be small at small $P$. For example, for $M_{p}=0.1 M_{\mathrm{J}}$ and $P=1$ day we find $\left\langle\tau_{E}\right\rangle \simeq 800 \mathrm{Myr}$. At sufficiently small $M_{p}$ and/or large $P$, the nonlinear effects 

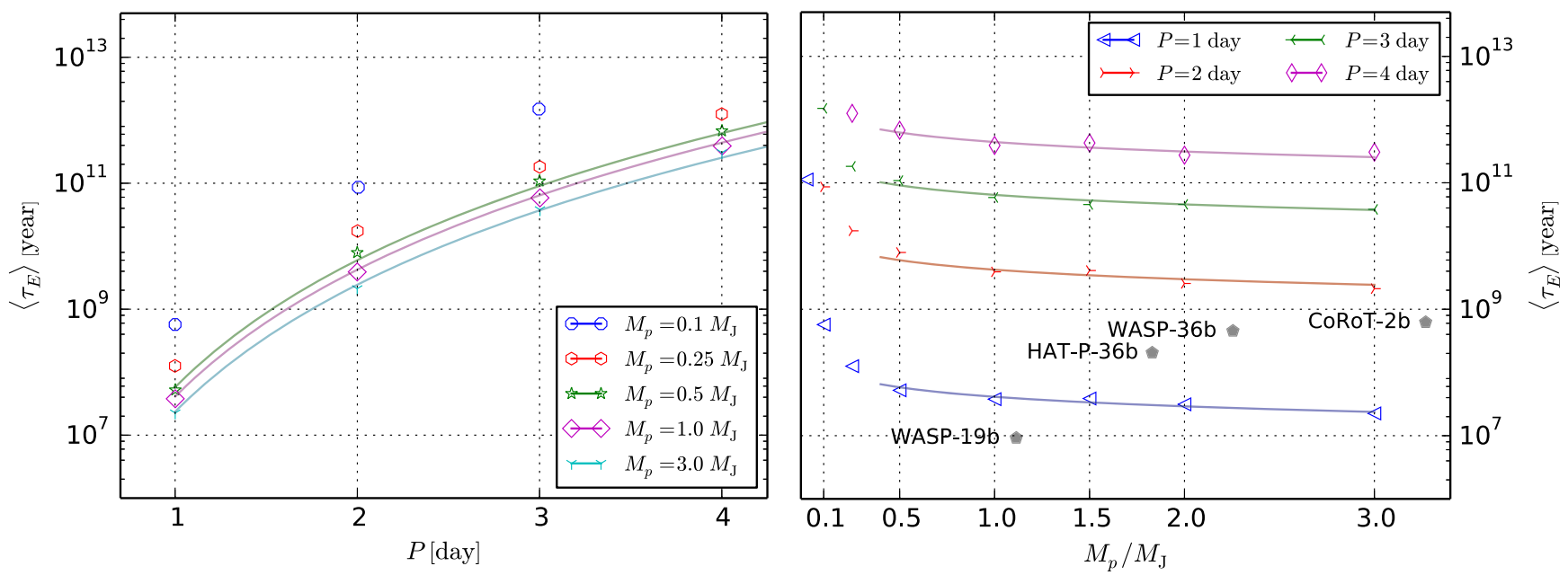

Figure 8. Dependence of $\left\langle\tau_{E}\right\rangle$ on $P$ for fixed values of $M_{p}$ (left panel) and the dependence of $\left\langle\tau_{E}\right\rangle$ on $M_{p}$ for fixed values of $P$ (right panel). The solid lines show our analytic fitting formula, which begins to break down for $M_{p} \lesssim 0.5 M_{\mathrm{J}}$.

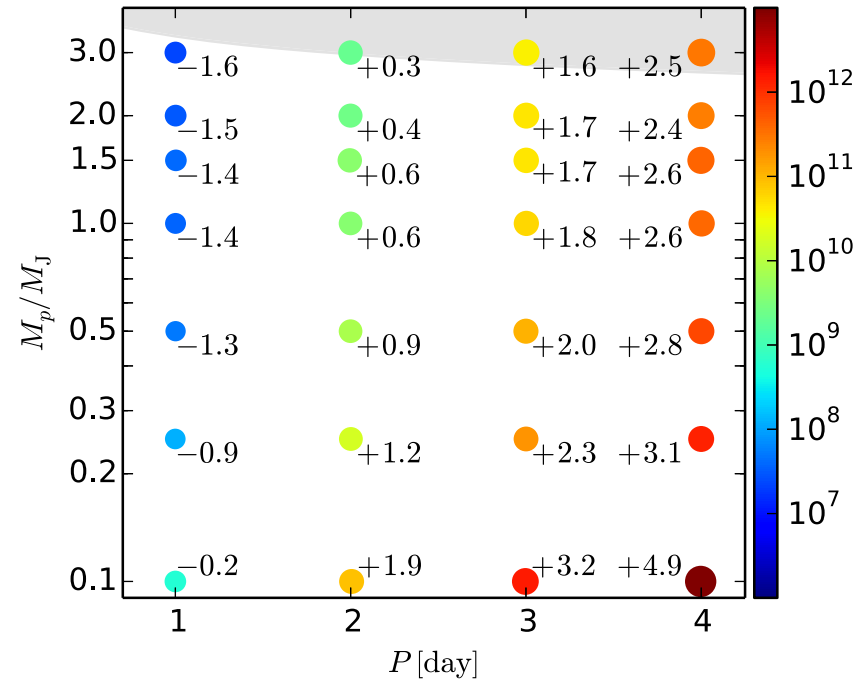

Figure 9. Decay time $\left\langle\tau_{E}\right\rangle$ on the $\left(M_{p}, P\right)$ plane. The size of the marker is proportional to $\log _{10}\left(Q_{*}^{\prime}\right)$, with corresponding color bar for $\left\langle\tau_{E}\right\rangle$ in years. Each sample point is labeled with $\log _{10}\left(\left\langle\tau_{E}\right\rangle / \mathrm{Gyr}\right)$. The shaded region represents Barker \& Ogilvie (2011)'s prediction for when linearly resonant modes break.

"turn off" and $\left\langle\tau_{E}\right\rangle$ collapses onto the linear result. This is particularly evident for $M_{p}=0.1 M_{\mathrm{J}}$ in Figure 8 . We also illustrate this effect in Figure 10, which shows how $\left\langle\tau_{E}\right\rangle$ depends on $P$ when $M_{p}=M_{\mathrm{J}}$ for different numbers of generations. We see that by $P \simeq 10$ days, $\left\langle\tau_{E}\right\rangle$ is very long and close to the linear prediction (the $N_{\text {gen }}=1$ line).

\subsection{Implications for a Few Known Systems}

Based on the Exoplanet Orbit Database (http://www. exoplanets.org), there are currently 11 known planets orbiting approximately solar-type stars $\left(M=1.0 \pm 0.1 M_{\odot}\right.$ and $T_{\text {eff }} \simeq 5500 \mathrm{~K}$ ) with decay times $\left\langle\tau_{E}\right\rangle<1 \mathrm{Gyr}$ according to our results. Of these, 7 have expected decay times $\left\langle\tau_{E}\right\rangle \lesssim 0.3 \mathrm{Gyr}$; in order of increasing $P$, they are WASP19b, TrES-3b, HAT-P-36b, WASP-77Ab, WASP-4b, WASP$36 \mathrm{~b}$, and WASP-46b. Figure 11 shows these planets on the $M_{p} \sin i-P$ plane, with $\left\langle\tau_{E}\right\rangle$ labeled for each system and a contour of $\left\langle\tau_{E}\right\rangle$ superimposed. These planets all have

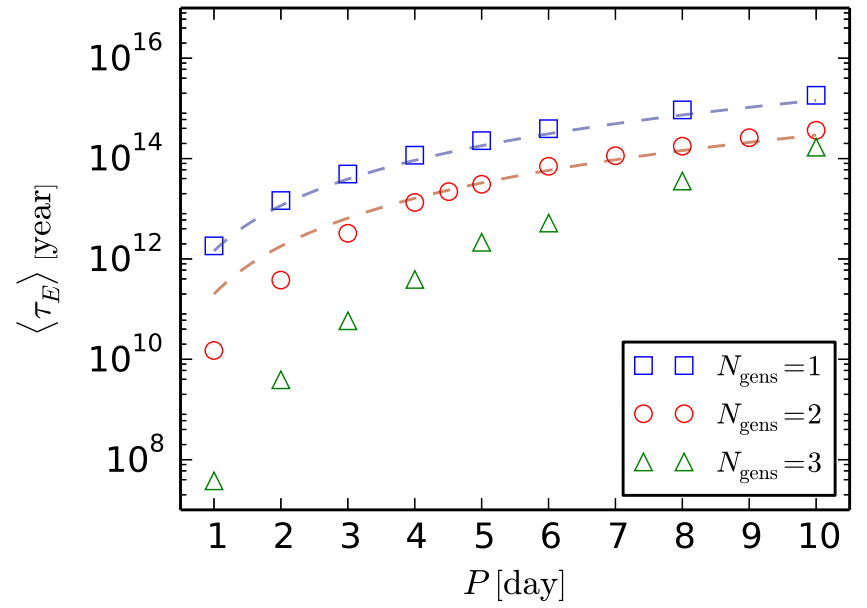

Figure 10. Decay time $\left\langle\tau_{E}\right\rangle$ as a function of $P$ for different numbers of generations and $M_{p}=M_{\mathrm{J}}$. (blue squares) 10 most resonant parents. (red circles) parents+daughters. (green triangles) parents+daughters+granddaughters. (dashed lines) analytic estimates for $N_{\text {gens }}=1,2$ (Appendix G).

$M_{p} \sin i>M_{\mathrm{J}}, P<2.0$ days, and eccentricities consistent with or very close to zero. Since these are all transiting systems, $M_{p} \sin i \simeq M_{p}$ and the reported errors in the measured mass are typically $\lesssim 0.1 M_{\mathrm{J}}$.

We note that two of the planets (CoRoT-2b and CoRoT-18b) have masses $M_{p} \sin i>3 M_{\mathrm{J}}$. This suggests that they are in the strongly nonlinear regime where the parent wave breaks within the stellar core (Barker \& Ogilvie 2010, 2011; Barker 2011). We discuss how our results compare to the strongly nonlinear simulations of Barker \& Ogilvie in Section 6.

Of the 11 planets with $\left\langle\tau_{E}\right\rangle<1 \mathrm{Gyr}$, there are five for which studies report at least some constraint on the age of the system. In three of these, the age uncertainties are sufficiently large that the systems might be relatively young, i.e., $\sim 1 \mathrm{Gyr}$ (WASP64b, WASP-5b, CoRoT-2b: $1.2_{-0.7}^{+1.2}, 5.4_{-4.3}^{+4.4}, 2.7_{-2.7}^{+3.2}$, respectively). However, WASP-4b and WASP-19b appear to be older systems: $7.0 \pm 2.9$ and $10.2_{-3.8}^{+3.0} \mathrm{Gyr}$, respectively. Assuming that the planets arrived close to their current orbits when their host stars first formed, such old stellar ages seem to be in tension with the small $\left\langle\tau_{E}\right\rangle$ we predict, especially in the case of WASP-19b. If our results are correct, then perhaps these 


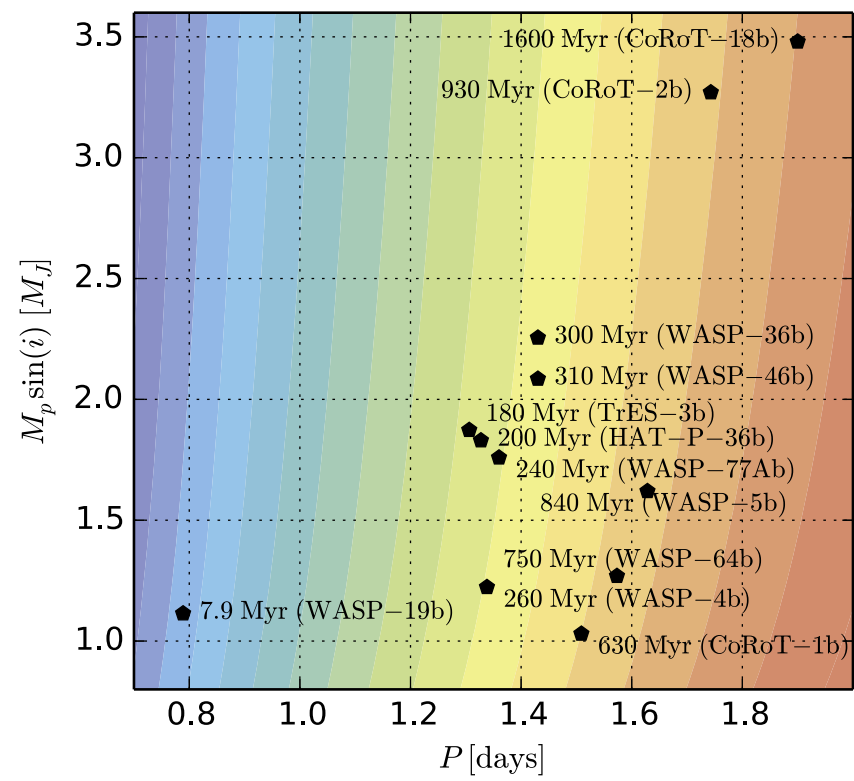

Figure 11. Period-mass distribution ( $M_{p} \sin i$ vs. orbital period $P$ ) of known extrasolar planets orbiting solar-type stars with $\left\langle\tau_{E}\right\rangle \lesssim 1 \mathrm{Gyr}$ based on our results. Each planet is labeled by our $\left\langle\tau_{E}\right\rangle$ fitting formula (Equation (25)), and the color represents contours of $\left\langle\tau_{E}\right\rangle$. (Data taken from http://www. exoplanets.org).

planets were scattered into their current orbits well after the stars formed or they just happened to initially reside at separations with decay timescales very close to their current ages.

Several recent papers consider the prospects for the direct detection of orbital decay of individual planets by measuring transit timing variations (TTVs) over long time baselines ( $\Delta t \gtrsim 5$ year, see Watson \& Marsh 2010; Birkby et al. 2014; Gandolfi et al. 2014; Valsecchi \& Rasio 2014). In order to evaluate this possibility, we simulated four known systems spanning a variety of companion masses and orbital periods (but each with a solar-type host ${ }^{6}$ ), calculating their tidally induced TTV $\left(T_{\text {shift }}\right)$ and change in orbital period $(\Delta P)$ as a function of $\Delta t$. We compute these according to (see Birkby et al. 2014 for a derivation)

$$
\begin{gathered}
T_{\text {shift }} \approx \frac{1}{2} \frac{\dot{\Omega}}{\Omega}(\Delta t)^{2}=\frac{3}{4 \tau_{E}}(\Delta t)^{2}, \\
\Delta P \approx \dot{P} \Delta t=\frac{3}{2} \frac{P E_{\text {orb }}}{\dot{E}_{\text {orb }}} \Delta t=\frac{3 P}{2 \tau_{E}} \Delta t .
\end{gathered}
$$

In order of increasing orbital period, we analyze WASP-19b (Hebb et al. 2010; Hellier et al. 2011; Mortier et al. 2013), HAT-P-36b (Bakos et al. 2012), WASP-36b (Smith et al. 2012), and CoRoT-2b (Alonso et al. 2008; Gillon et al. 2010).

In order to calculate the orbital decay rate of these systems, we simulate a small range of orbital periods centered on each system's measured period. We then compute the time-averaged decay rates following the procedure described in Section 4.6. We do this in order to mitigate any differences between the resonances of our stellar models and the actual resonances of the stellar hosts. Furthermore, this allows us to compute a

\footnotetext{
6 This requirement is why we do not consider WASP-18b, which was analyzed in Birkby et al. (2014).
}

minimum and maximum expected decay rate, corresponding to the troughs and peaks of the resonances, respectively.

Table 1 lists $\left\langle\tau_{E}\right\rangle$ as well as the minimum and maximum $\tau_{E}$. The $\left\langle\tau_{E}\right\rangle$ of the four systems ranges from about $10 \mathrm{Myr}$ (WASP-19b) to $600 \mathrm{Myr}$ (CoRoT-2b), while the minimum (maximum) $\tau_{E}$ is approximately two times smaller (larger). WASP-19b has by far the shortest decay time owing to its extremely short orbital period (18.9 hr).

Table 1 also lists the systems' average, minimum, and maximum $\Delta P$ and $T_{\text {shift }}$ after 10 years of evolution. These provide an estimate of the magnitude of the tidally induced deviations we would expect to observe from these systems over the next 10 years.

We quantify these effects further in Figure 12, which shows the cumulative probability of observing tide-induced deviations as a function of time. We choose a detection threshold of $(\Delta P)_{\mathrm{thr}}=0.1 \mathrm{~s}$ and $\left(T_{\mathrm{shift}}\right)_{\mathrm{thr}}=60 \mathrm{~s}$ based on the measurement errors of $P$ and the expected uncertainties in TTVs (Gillon et al. 2009; Watson \& Marsh 2010); different choices will scale $\Delta t$ through Equation (27).

We find that $T_{\text {shift }}$ should always produce a detection faster than $\Delta P$. This is because $T_{\text {shift }}$ is a cumulative effect that builds up throughout the orbital decay. According to our results, WASP-19b should produce a detectable $T_{\text {shift }}$ in the very near future, with $a \approx 50 \%$ chance of observing a deviation now given the current four year baseline (Hellier et al. 2011) and a high likelihood of detection after only two more years. It will take considerably longer before detections are possible in the other three systems.

We note that even if, for some reason, our calculations overestimate the dissipation rate by an order of magnitude, the $T_{\text {shift }}$ curves in Figure 12 would only be shifted to the right by a factor of $\sqrt{10} \sim 3$. Finally, as Watson \& Marsh (2010) point out, the Applegate effect could produce $\Delta P$ and $T_{\text {shift }}$ values that are comparable to the tidally induced values and distinguishing the two may not be simple.

\subsection{Comparison with Previous Estimates of Nonlinear Tidal Dissipation}

Previous studies that attempt to estimate the nonlinear dissipation rate of dynamical tides in close binaries include Kumar \& Goodman (1996) and Barker \& Ogilvie (2011). They both argue that an upper bound to the dissipation rate is approximately given by the product of the parent's linear energy and the three-mode growth rate of the fastest growing daughter pair:

$$
\dot{E}_{*} \lesssim \Gamma_{3 \mathrm{md}} E_{\text {lin }}
$$

This estimate does not account for the continuous linear driving of the parent. Instead, the parent wave is initialized with an energy equal to $E_{\text {lin }}$ but is otherwise undriven, and the problem reduces to determining the amount of time it takes for daughters to dissipate that initial energy. Although this is appropriate for the tidal capture problem that Kumar \& Goodman (1996) consider (because the binary is on a highly eccentric orbit and the parent is only driven strongly during the brief pericenter passage), in our analysis the orbit is circular and the parent is a continuously driven standing wave. The estimate of Equation (28) also assumes that the mode dynamics are dominated by the single, fastest growing daughter pair even 
Table 1

Orbital Parameters for Known Systems and a Summary of Predictions for the Orbital Decay Timescales and Change in Orbital Parameters after 10 Years

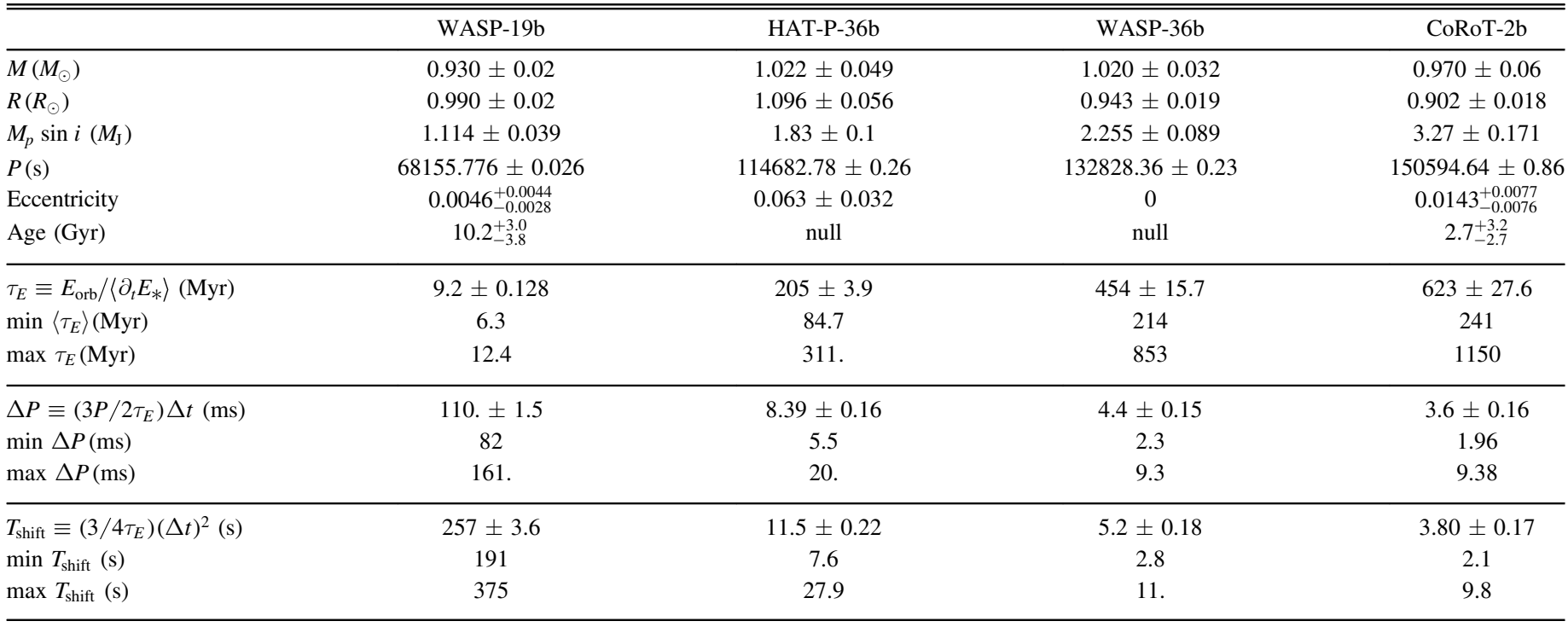
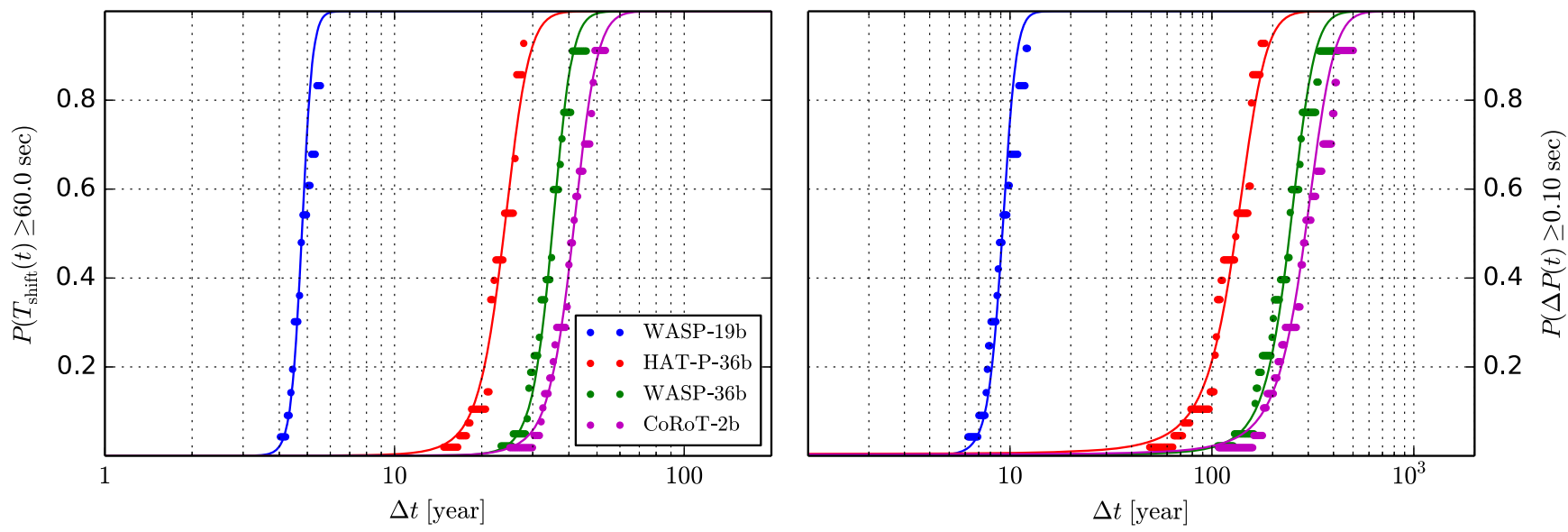

Figure 12. Cumulative probability distributions for observable changes as a function of elapsed time. (blue) WASP-19b. (red) HAT-P-36b. (green) WASP-36b. (purple) CoRoT-2b. Solid lines represent approximate fits to the simulation results, which are represented by filled circles.

though there may be many modes participating in the interactions.

Using Equation (17), we find that the fastest growing daughters have a growth rate of

$$
\Gamma_{3 \mathrm{md}} \simeq 0.6\left(\frac{M_{p}}{M_{\mathrm{J}}}\right)\left(\frac{P}{\text { day }}\right)^{-11 / 6} \mathrm{yr}^{-1}
$$

and with Equation (15)

$$
\Gamma_{3 \mathrm{md}} E_{\mathrm{lin}} \simeq 2.4 \times 10^{28}\left(\frac{M_{p}}{M_{\mathrm{J}}}\right)^{3}\left(\frac{P}{\mathrm{day}}\right)^{-7.5} \mathrm{erg} \mathrm{s}^{-1} .
$$

For comparison, the fit to our numerical simulations yields, by Equations (12) and (25),

$$
\dot{E}_{*} \simeq 3.5 \times 10^{29}\left(\frac{M_{p}}{M_{\mathrm{J}}}\right)^{1.5}\left(\frac{P}{\text { day }}\right)^{-7.4} \mathrm{erg} \mathrm{s}^{-1} .
$$

Thus, while the two have nearly identical $P$ scalings, the $\dot{E}_{*}$ from our simulations is $\simeq 15$ times larger than $\Gamma_{3 \mathrm{md}} E_{\text {lin }}$ for $M_{p} \simeq M_{\mathrm{J}}$. This factor of 15 difference can be seen in the $Q_{*}^{\prime}$ estimates. In particular, we find $Q_{*}^{\prime} \approx 3 \times 10^{5}$ at $P=1$ day for $M_{p} \gtrsim 0.5 M_{\mathrm{J}}$. By contrast, Barker \& Ogilvie (2010) argue that $Q_{*}^{\prime} \gtrsim 5 \times 10^{6}$ for systems below the wave breaking limit $\left(M_{p} \lesssim 3 M_{\mathrm{J}}\right)$ based on their assumption that $\dot{E}_{*} \lesssim \Gamma_{3 \mathrm{md}} E_{\text {lin }}$ in the weakly nonlinear regime.

We suspect that the discrepancy is largely due to the assumption in Equation (28) that only the single fastest growing daughter pair is important. In Figure 13 we demonstrate that this is not the case. We show the individual and cumulative contribution to $\dot{E}_{*}$ of modes in our reference network (which consists of $\simeq 1500$ modes). We find that there are several daughter modes that contribute substantial amounts of dissipation, not just a single dominant daughter pair. Figure 13 also shows that, in sum, the granddaughters are the dominant source of dissipation in the network.

\section{SUMMARY AND DISCUSSION}

We present a first principles calculation of the saturation of nonlinear interactions between $g$-modes excited within the cores of solar-type hosts by planetary companions. Using a 


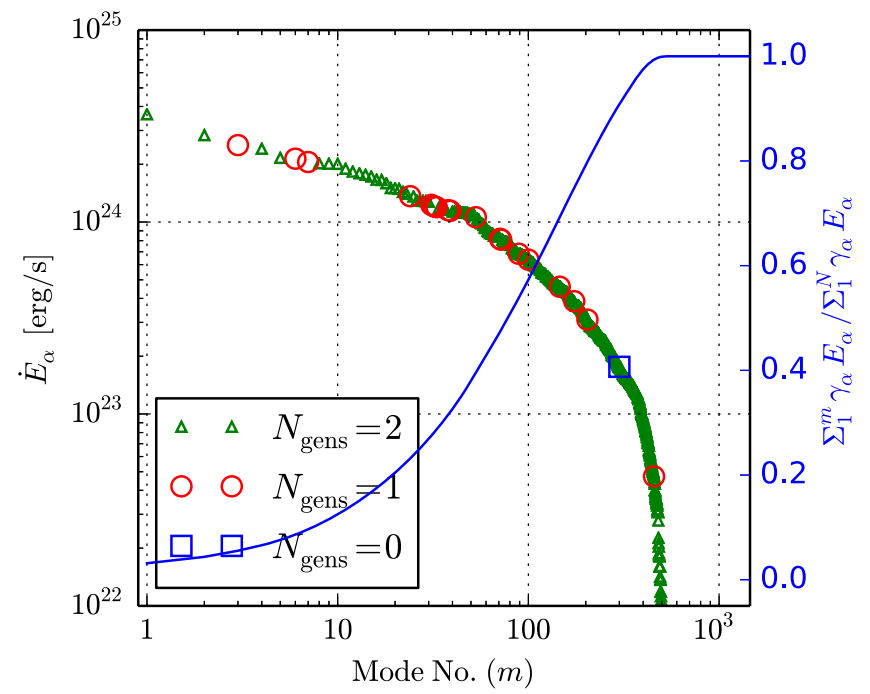

Figure 13. Energy dissipation rate $\dot{E}_{\alpha}$ for each mode in our reference network, which consists of one parent (blue square), $\simeq 20$ daughters (red circles) and $\simeq 1500$ granddaughters (green triangles). Modes are ordered by $\dot{E}_{\alpha}$ and here we take $M_{p}=M_{\mathrm{J}}$ and $P \simeq 3$ days. The blue line is the cumulative distribution of $\dot{E}_{\alpha}$; we see that granddaughter modes are responsible for the majority of the dissipation.

WKB approximation for high-order, adiabatic $g$-modes and analytic approximations to their coupling coefficients detailed in WAQB, we systematically investigate the number of modes and types of couplings that are dynamically relevant. We determine the minimum mode network size and structure that yields total dissipation rates consistent with those of much larger networks (to within a factor of $\approx 2$ ). This minimum network is sufficiently nimble that we can efficiently explore broad swaths of the $\left(M_{p}, P\right)$-plane. We find that weakly nonlinear interactions are energetically important over large portions of this plane, including regions occupied by known exoplanetary systems. In these regions, the orbital decay time $\left\langle\tau_{E}\right\rangle$ and stellar tidal quality factor $Q_{*}^{\prime}$ follow simple power-law relations (Equations (25) and (26)).

We find that the orbital decay of a number of observed hot Jupiters should occur on timescales much shorter than the main-sequence lifetime of their host star. Such rapid orbital decay could explain the observed paucity of giant planets with $P \lesssim 2$ days (McQuillan et al. 2013; see also Winn \& Fabrycky 2015 for a recent review of the observations). The short decay times would also induce TTVs that may be observable with current technology (especially that of WASP19b). Precision photometry of individual systems may thus provide a new handle on tidal interactions within the next few years.

Our calculation comes with some caveats. First, although our reference network yields dissipation results that are very similar to those of the largest networks we investigate (which have $\gtrsim 10$ times more modes than the reference network), there is still a possibility that the dynamics will change upon the addition of even more modes. Second, our calculation assumes that the modes are all global standing waves. However, this prescription may break down if the amplitudes of the modes change on timescales shorter than the group travel times between their inner and outer turning points. Moreover, although the parent mode is below the wave breaking threshold (when not too close to a linear resonance), the daughter and granddaughter modes may not be. In Appendix F, we show that the threshold amplitude of the three-mode parametric instability is much smaller than the wave breaking threshold and that both have the same frequency scaling. This may mean that further generations will be excited before the daughter and granddaughter modes break. Nonetheless, this issue deserves further investigation. Finally, we do not account for possible changes to the stellar structure due to the transfer of energy and angular momentum from the sea of excited waves. Further work is needed in order to determine the extent to which stellar spin-up, heating, and/or evolution affect background properties such as the star's stratification and thereby the wave interaction dynamics (see Barker \& Ogilvie 2010 for a discussion of this issue).

Our study focuses on wave interactions in the weakly nonlinear regime. For solar type stars, this corresponds to planetary masses $M_{p} \lesssim 3.6 M_{\mathrm{J}}(P / 1 \text { day })^{-0.1}$; above this mass, the parent wave breaks as it approaches the stellar center and the system is therefore in the strongly nonlinear regime (Barker \& Ogilvie 2010, 2011; Barker 2011). In the weakly nonlinear regime the parent is a global standing wave while in the strongly nonlinear regime the parent is more appropriately treated as a traveling wave; it does not reflect upon reaching the stellar center. Barker \& Ogilvie study the fate of such a strongly nonlinear traveling wave with numerical simulations using a Boussinesq-type model. Because our calculation studies a different hydrodynamic regime, a direct comparison with their results is not possible. Nonetheless, one might expect the two to roughly agree near the region that marks the transition from weakly nonlinear to strongly nonlinear (i.e., near $M_{p} \simeq 3 M_{\mathrm{J}}$ ). Indeed, Barker (2011) finds $Q_{*}^{\prime} \approx 9 \times 10^{4}(P / \text { day })^{2.8}$ for waves that break while we find $Q_{*}^{\prime} \approx 5 \times 10^{5}(P / \text { day })^{2.4}$ for $M_{p} \simeq 3 M_{\mathrm{J}}$. We explore some of the similarities between the two regimes further in Appendix F.

We find $Q_{*}^{\prime} \approx 3 \times 10^{5}$ at $P=1$ day for $M_{p} \gtrsim 0.5 M_{\mathrm{J}}$. This appears to conflict with the estimate in Barker \& Ogilvie (2010), who argue that $Q_{*}^{\prime}$ increases rapidly to $Q_{*}^{\prime} \gtrsim 5 \times 10^{6}$ for systems below the wave breaking limit $\left(M_{p} \lesssim 3 M_{\mathrm{J}}\right)$. They do not attempt to calculate the saturation of the nonlinear parametric instabilities as in our study but instead base their estimate on stability analysis scaling arguments. As we explain in Section 5.2, the issue might be that their estimate neglects the continuous driving of the parent and does not account for the complicated multi-mode dynamics that we find are important. Interestingly, we do see a steep increase in $\left\langle\tau_{E}\right\rangle$, although at much lower $M_{p}$.

In order to be consistent with the observed distribution of exoplanets, Penev et al. (2012) find that $Q_{*}^{\prime} \gtrsim 10^{7}$. However, as Birkby et al. (2014) note, their analysis is for one specific set of initial conditions with some idealized assumptions about the chances of a planet candidate being confirmed by follow-up. They also assume gas disk migration and, as Penev et al. (2012) point out, their result may not be valid for other giant planet migration mechanisms such as dynamical scattering. If gas migration is the dominant mechanism that creates hot Jupiters, then our results suggest that finding these systems at $P \lesssim 2$ days should be extremely rare. However, if scattering populates short period orbits at random times after a system's formation, then a low $Q_{*}^{\prime}$ may not necessarily conflict with the observed population of hot Jupiters orbiting $\sim$ Gyr old hosts.

Our study only considers solar-type hosts even though the observed population of hot Jupiters includes a wide variety of 
host types. Since the linear and nonlinear excitation of waves by the tide is sensitive to the detailed structure of the star, it is not clear how our results might depend on stellar type. Extending the analysis to non-solar type hosts would therefore allow us to more fully assess the prospects for measuring tideinduced orbital decay of individual hot Jupiter systems.

We thank Phil Arras and the referee for valuable comments on this manuscript. R.E. is supported in part by the National Science Foundation and the LIGO Laboratory (PHY-0757058). This work was also supported by NASA NNX14AB40G.

\section{APPENDIX A COMPUTATION OF TIME-AVERAGES}

Quantities such as the energy dissipation rate $\dot{E}_{*}$ depend on how close the system happens to be to the densely spaced linear resonance peaks (the frequency spacing is $\left.\left|\Delta_{a}\right| / \Omega \approx 10^{-2}(P / \text { day })^{-1}\right)$. Because we are mostly interested in time-averaged statistics, at each $\left(M_{p}, P\right)$ point, we carry out 21 distinct simulations, each separated slightly in orbital period with a spacing chosen such that they span three resonance peaks (see Section 4.6 and Figure 2). We compute the timeaveraged statistic of a quantity $X$ by weighting each sample by the amount of time spent at that period

$$
\langle X\rangle=\frac{\int d t X}{\int d t}=\frac{\int d P \dot{P}^{-1} X}{\int d P \dot{P}^{-1}},
$$

where $\dot{P}=d P / d t$ is the rate at which the period changes due to tidal dissipation. We compute $\dot{P}$ using an energy-balance argument. We expect the time rate-of-change of the orbital energy $\left(E_{\text {orb }}\right)$, the tidal interaction-energy $\left(E_{\text {int }}\right)$, the rotational energy of the synchronized companion $\left(E_{\mathrm{rot}}\right)$, and the energy stored in the modes $\left(E_{\text {modes }}\right)$ to balance with the energy lost through dissipation

$$
\frac{d}{d t}\left(E_{\mathrm{orb}}+E_{\mathrm{int}}+E_{\mathrm{rot}}+E_{\mathrm{modes}}\right)=-2 \sum_{i} \gamma_{i} E_{i}
$$

from which we can extract the time rate-of-change of the orbital period via

$$
\begin{aligned}
& \dot{P}=-2\left(\frac{d E_{\text {orb }}}{d P}+\right.\left.\frac{d E_{\text {int }}}{d P}+\frac{d E_{\text {rot }}}{d P}+\frac{d E_{\text {modes }}}{d P}\right)^{-1} \sum_{i} \gamma_{i} E_{i} \\
& \approx-2\left(\frac{d E_{\text {orb }}}{d P}\right)^{-1} \sum_{i} \gamma_{i} E_{i} \\
& \propto \frac{P}{E_{\text {orb }}} \sum_{i} \gamma_{i} E_{i},
\end{aligned}
$$

where we have noted that $\left|d E_{\text {orb }} / d P\right| \gg\left|d E_{\text {int }} / d P\right|$, $\left|d E_{\text {rot }} / d P\right|,\left|d E_{\text {modes }} / d P\right|$. This is because $E_{\text {orb }}$ is much larger than any of the other energy scales, so even small relative changes in $E_{\text {orb }}$ dominate over the other terms.
This gives

$$
\langle X\rangle=\frac{\int d P\left(\frac{P}{E_{\mathrm{orb}}} \sum_{i} \gamma_{i} E_{i}\right)^{-1} X}{\int d P\left(\frac{P}{E_{\mathrm{orb}}} \sum_{i} \gamma_{i} E_{i}\right)^{-1}} \approx \frac{\sum_{P}\left(\frac{P}{E_{\mathrm{orb}}} \sum_{i} \gamma_{i} E_{i}\right)^{-1} X}{\sum_{P}\left(\frac{P}{E_{\mathrm{orb}}} \sum_{i} \gamma_{i} E_{i}\right)^{-1}} .
$$

We use this procedure to calculate the time-averaged $\dot{E}_{*}$ in the neighborhoods of each orbital period and $\left\langle\tau_{E}\right\rangle$.

\section{APPENDIX B \\ THREE-MODE NONLINEAR EQUILIBRIUM}

Here we briefly review the nonlinear equilibrium for threemode systems. The calculation is similar to that of Appendix D of WAQB except that here we provide more detail about the phase relations amongst the modes. We begin with the equations of motion (Equation (6)) and introduce the change of coordinates $x=q e^{-i(\omega-\Delta) t}$, yielding

$$
\begin{gathered}
\partial_{t} x_{\alpha}+\left(i \Delta_{\alpha}+\gamma_{\alpha}\right) x_{\alpha}=i \omega_{\alpha} U_{\alpha} e^{-i\left(m_{\alpha} \Omega-\omega_{\alpha}+\Delta_{\alpha}\right) t} \\
+2 i \omega_{\alpha} k_{\alpha \beta \gamma} x_{\beta}^{*} x_{\gamma}^{*} e^{i\left(\omega_{\alpha}+\omega_{\beta}+\omega_{\gamma}-\Delta_{\alpha}-\Delta_{\beta}-\Delta_{\gamma}\right) t} \\
\partial_{t} x_{\beta}+\left(i \Delta_{\beta}+\gamma_{\beta}\right) x_{\beta} \\
=2 i \omega_{\beta} k_{\alpha \beta \gamma} x_{\alpha}^{*} x_{\gamma}^{*} e^{i\left(\omega_{\alpha}+\omega_{\beta}+\omega_{\gamma}-\Delta_{\alpha}-\Delta_{\beta}-\Delta_{\gamma}\right) t} \\
\partial_{t} x_{\gamma}+\left(i \Delta_{\gamma}+\gamma_{\gamma}\right) x_{\gamma} \\
=2 i \omega_{\gamma} k_{\alpha \beta \gamma} x_{\alpha}^{*} x_{\beta}^{*} e^{i\left(\omega_{\alpha}+\omega_{\beta}+\omega_{\gamma}-\Delta_{\alpha}-\Delta_{\beta}-\Delta_{\gamma}\right) t} .
\end{gathered}
$$

We can cancel all time dependence in these equations by demanding

$$
m_{\alpha} \Omega=\omega_{\alpha}-\Delta_{\alpha}=\Delta_{\beta}+\Delta_{\gamma}-\omega_{\beta}-\omega_{\gamma}
$$

and assuming that $\partial_{t} \rightarrow 0$ in order to explicitly seek time independent solutions. Manipulating the two daughter equations yields

$$
\begin{aligned}
\left(i \Delta_{\beta}+\gamma_{\beta}\right) x_{\beta}\left(-i \Delta_{\gamma}+\gamma_{\gamma}\right) & =2 i \omega_{\beta} k_{\alpha \beta \gamma} x_{\alpha}^{*}\left(\left(i \Delta_{\gamma}+\gamma_{\gamma}\right) x_{\gamma}\right)^{*} \\
& =4 \omega_{\beta} \omega_{\gamma} k_{\alpha \beta \gamma}^{2} x_{\alpha} x_{\alpha}^{*} x_{\beta}
\end{aligned}
$$

which implies

$$
\Delta_{\beta} \gamma_{\gamma}=\Delta_{\gamma} \gamma_{\beta}, \Delta_{\beta} \Delta_{\gamma}+\gamma_{\beta} \gamma_{\gamma}=4 \omega_{\beta} \omega_{\gamma} k_{\alpha \beta \gamma}^{2} A_{\alpha}^{2},
$$

where we write $x=A e^{i \delta}$. We then have

$$
A_{\alpha}^{2}=\frac{\Delta_{\beta} \Delta_{\gamma}+\gamma_{\beta} \gamma_{\gamma}}{4 \omega_{\beta} \omega_{\gamma} k_{\alpha \beta \gamma}^{2}}=\frac{\gamma_{\beta} \gamma_{\gamma}}{4 \omega_{\beta} \omega_{\gamma} k_{\alpha \beta \gamma}^{2}}\left[1+\left(\frac{\Delta_{\beta}+\Delta_{\gamma}}{\gamma_{\beta}+\gamma_{\gamma}}\right)^{2}\right]
$$

and we recover the parent instability threshold energy $E_{\mathrm{thr}}=A_{\alpha}^{2}$ (Equation (16)). The daughter equations yield

$$
\frac{\left(i \Delta_{\beta}+\gamma_{\beta}\right) x_{\beta}}{\omega_{\beta} x_{\gamma}}=\frac{\left(i \Delta_{\gamma}+\gamma_{\gamma}\right) x_{\gamma}}{\omega_{\gamma} x_{\beta}} \Longrightarrow\left(\frac{A_{\beta}}{A_{\gamma}}\right)^{2}=\frac{\gamma_{\gamma} \omega_{\beta}}{\gamma_{\beta} \omega_{\gamma}},
$$

which gives

$$
\left(i \Delta_{\beta}+\gamma_{\beta}\right) A_{\beta}=2 i \omega_{\beta} k_{\alpha \beta \gamma} A_{\alpha} A_{\gamma} e^{-i\left(\delta_{\alpha}+\delta_{\beta}+\delta_{\gamma}\right)},
$$


or equivalently

$$
\gamma_{\beta} \frac{A_{\beta}}{A_{\gamma}}=2 \omega_{\beta} k_{\alpha \beta \gamma} A_{\alpha} \sin \delta, \Delta_{\beta} \frac{A_{\beta}}{A_{\gamma}}=2 \omega_{\beta} k_{\alpha \beta \gamma} A_{\alpha} \cos \delta,
$$

where $\delta=\delta_{\alpha}+\delta_{\beta}+\delta_{\gamma}$. We can now use the parent equation to determine the parent phase $\delta_{\alpha}$ and the product of the daughter amplitudes

$$
\left(i \Delta_{\alpha}+\gamma_{\alpha}\right) A_{\alpha}=i \omega_{\alpha} U_{\alpha} e^{-i \delta_{\alpha}}+2 i \omega_{\alpha} k_{\alpha \beta \gamma} A_{\beta} A_{\gamma} e^{-i \delta} .
$$

After some manipulation, we find

$$
\begin{aligned}
& A_{\beta} A_{\gamma} \\
& =\left(\frac{A_{\alpha}}{2 \omega_{\alpha} \kappa_{\alpha \beta \gamma}}\right)\left[\left(\Delta_{\alpha} \cos \delta+\gamma_{\alpha} \sin \delta\right)\right. \\
& \left. \pm \sqrt{\left(\Delta_{\alpha} \cos \delta+\gamma_{\alpha} \sin \delta\right)^{2}+\frac{\Delta_{\alpha}^{2}+\gamma_{\alpha}^{2}}{A_{\alpha}^{2}}\left(\frac{\omega_{\alpha}^{2} U_{\alpha}^{2}}{\Delta_{\alpha}^{2}+\gamma_{\alpha}^{2}}-A_{\alpha}^{2}\right)}\right]
\end{aligned}
$$

The choice of sign depends on the sign of $\omega_{\alpha} \kappa_{\alpha \beta \gamma}$ and is determined by the requirement that the daughter amplitudes be positive. Finally, by Equation (15), we see that the instability condition is

$$
\frac{\omega_{\alpha}^{2} U_{\alpha}^{2}}{\Delta_{\alpha}^{2}+\gamma_{\alpha}^{2}}=\frac{E_{\mathrm{lin}}}{E_{0}}>A_{\alpha}^{2}=\frac{E_{\mathrm{thr}}}{E_{0}} .
$$

Note that we can solve for the parent's phase $\delta_{\alpha}$ and the sum of all the mode phases $\delta$, but we cannot break the degeneracy between the daughters' phases. This is observed numerically, and carries information about the initial conditions.

\section{APPENDIX C TWO DAUGHTERS, N PARENTS}

If we linearize around the linear-equilibrium solution, the equations of motion for the daughter modes become

$$
\partial_{t} q_{\beta}+\left(i \omega_{\beta}+\gamma_{\beta}\right)=2 i \omega_{\beta} q_{\gamma}^{*} \sum_{p \in \text { parents }} \kappa_{p \beta \gamma} q_{p}^{*}
$$

for daughter $\beta$ and the equivalent equation with the exchange $\gamma \leftrightarrow \beta$ for daughter $\gamma$. We can analyze this system as if there is a single parent with complex amplitude

$$
\kappa q=\sum_{p \in \text { parents }} \kappa_{p \beta \gamma} q_{p}^{*} .
$$

We note the possibility for parent modes to interfere with one another when driving daughter modes, possibly rendering daughters stable under multi-parent driving when they were unstable to any individual parent. Most notably, if the parents are nearly regularly spaced in frequency and driven at the midpoint between their resonance peaks, there can be strong destructive interference. This is because each parent is paired with a partner on the opposite side of the driving frequency, and each pair consists of parents oscillating with nearly opposite phases. This narrow "trap" in the resonance troughs is readily apparent at orbital periods above 4 days for a solar-type host of a Jupiter mass companion. However, we did not observe significant "trapping" below $\sim 4$ day orbital periods, where we focus our attention for this study. This may be due to the asymmetric spacing of resonances, which will destroy this near perfect cancellation, or due to the amplitudes being large enough to overcome any cancellation that was present. In the hot Jupiter context, this issue is probably only of theoretical interest since the orbital evolution timescales are $\gtrsim 10^{11}$ year for $P \gtrsim 3$ day, even for a $3 M_{\mathrm{J}}$ companion.

\section{APPENDIX D \\ DETAILS OF COLLECTIVE SET SELECTION ALGORITHM}

One can easily think of more complicated collective sets than what is described in Section 3.2. We analyze several of these systems in Appendix E. In order to detect and include the diverse set of collective systems, we implement a broad search through parameter space. We begin with a "seed" triple in parameter space, typically taken to be a minima of $E_{\mathrm{thr}}$. We then expand the set of included modes in $(n, l)$-space around these seeds, choosing new modes from the border of the included set. For these border modes, we compute the threemode $E_{\text {thr }}$ for all possible couplings between that border mode and the interior modes. We then sort these $E_{\mathrm{thr}}$, and divide each by the number of couplings that produce $E_{\mathrm{thr}}$ less than or equal to the current value. We take the minimum ratio and call it the "collective $E_{\text {thr." }}$ "This approximates the scaling with $N$ predicted in Appendix E and incorporates the decoupling of large detuning modes discussed in Appendix E.4. Border modes are added in order of increasing collective $E_{\mathrm{thr}}$, and these thresholds are updated each time a mode is added to the network. If the detuning increases the three-mode $E_{\text {thr }}$ faster than the number of modes included, then small sets with low detuning will naturally be chosen. However, if the detuning increases $E_{\text {thr }}$ more slowly than the number of modes included, then the collective $E_{\mathrm{thr}}$ will decrease with the addition of more modes and the algorithm will select a set of collectively unstable daughters.

We typically find that a minimum number of daughters is needed before the scaling with $N$ dominates over the increase in $E_{\text {thr. }}$. Depending on $E_{\text {lin }}$, these collective sets can grow to several thousand modes. Although each mode can only directly couple to a relatively small number of other modes (see Section 3.2 and WAQB), we find that many smaller sets overlap and are thereby strung together to create larger networks. We discuss some of this behavior in Section E.2.

This algorithm scales poorly with the number of modes included $\left(\mathrm{O}\left[\mathrm{N}^{3}\right]\right)$. Furthermore, as we describe in Section 4, we find that we can accurately model the total dissipation within the star using only three-mode pairs, rather than collective sets. This, coupled with the fact that large collective networks are expensive to integrate, is the reason we choose three-mode networks with many couplings and generations as our reference networks discussed in Sections 4.6, 5, and 5.1. We note, however, that collective sets may be important if one is interested in accurately modeling the dynamics of any particular mode, rather than the network as a whole.

\section{APPENDIX E COLLECTIVE SET STABILITY}

In this appendix we analyze collective instabilities, i.e., sets of daughter modes that display rapid growth rates due to their mutual inter-coupling. Although in our simulations we find that they do not contribute significantly to the total tidal dissipation 
in hot Jupiter systems, for completeness we present here derivations of different stability thresholds for different types of collective instabilities. Our mode selection algorithm (Appendix D) finds complicated collective sets that contain these types of coupling topologies.

\section{E.1. Single Collective Set}

We first consider the stability of a single collective set. Since we are interested in the stability of linear solutions, we can assume that the parent is at a fixed amplitude

$$
q_{o}=A_{o} e^{-i\left(\Omega t-\delta_{o}\right)}
$$

The equation of motion of each daughter is then

$$
\begin{gathered}
\dot{q}_{\alpha}+\left(i \omega_{\alpha}+\gamma_{\alpha}\right) q_{\alpha}=i \omega_{\alpha} \sum_{\beta} \kappa_{o \alpha \beta} A_{o} e^{+i\left(\Omega t-\delta_{o}\right)} q_{\beta}^{*} \\
=i \omega_{\alpha} \kappa_{o \alpha \alpha} A_{o} e^{+i\left(\Omega t-\delta_{o}\right)} q_{\alpha}^{*}+2 i \omega_{\alpha} \sum_{\beta \neq \alpha} \kappa_{o \alpha \beta} A_{o} e^{+i\left(\Omega t-\delta_{o}\right)} q_{\beta}^{*} .
\end{gathered}
$$

Defining a new set of variables $q=x e^{-i(\omega-\Delta) t}$, we can re-write the daughter equations as

$$
\begin{aligned}
\dot{x}_{\alpha}+ & \left(i \Delta_{\alpha}+\gamma_{\alpha}\right) x_{\alpha}=i \omega_{\alpha} \kappa_{o \alpha \alpha} A_{o} x_{\beta}^{*} e^{+i\left(\Omega+2 \omega_{\alpha}-2 \Delta_{\alpha}\right) t-i \delta_{o}} \\
& +2 i \omega_{\alpha} \sum_{\beta \neq \alpha} \kappa_{o \alpha \beta} A_{o} x_{\beta}^{*} e^{+i\left(\Omega+\omega_{\alpha}+\omega_{\beta}-\Delta_{\alpha}-\Delta_{\beta}\right) t-i \delta_{o}} \\
& =i \omega_{\alpha} \kappa_{o \alpha \alpha} A_{o} x_{\beta}^{*} e^{-i \delta_{o}}+2 i \omega_{\alpha} \sum_{\beta \neq \alpha} \kappa_{o \alpha \beta} A_{o} x_{\beta}^{*} e^{-i \delta_{o}}
\end{aligned}
$$

where in the last step we demanded that the time dependence cancels

$$
\Omega+\omega_{\alpha}+\omega_{\beta}-\Delta_{\alpha}-\Delta_{\beta}=0 \forall\{\alpha, \beta\} .
$$

Analyzing this as an eigenvalue problem, separate $x$ into real and imaginary parts $x=R+i I$,

$$
\begin{aligned}
& {\left[\begin{array}{c}
\dot{R}_{\alpha} \\
\dot{I}_{\alpha}
\end{array}\right]=} \\
& \quad\left[\begin{array}{ll}
-\gamma_{\alpha}+\omega_{\alpha} A_{o} \kappa_{o \alpha \alpha} \sin \delta_{o} & \Delta_{\alpha}+\omega_{\alpha} A_{o} \kappa_{o \alpha \alpha} \cos \delta_{o} \\
-\Delta_{\alpha}+\omega_{\alpha} A_{o} \kappa_{o \alpha \alpha} \cos \delta_{o} & -\gamma_{\alpha}-\omega_{\alpha} A_{o} \kappa_{o \alpha \alpha} \sin \delta_{o}
\end{array}\right] \\
& \quad \times\left[\begin{array}{c}
R_{\alpha} \\
I_{\alpha}
\end{array}\right] \\
& \quad+\sum_{\beta \neq \alpha}\left[\begin{array}{ll}
2 \omega_{\alpha} A_{o} \kappa_{o \alpha \beta} \sin \delta_{o} & 2 \omega_{\alpha} A_{o} \kappa_{o \alpha \beta} \cos \delta_{o} \\
2 \omega_{\alpha} A_{o} \kappa_{o \alpha \beta} \cos \delta_{o} & -2 \omega_{\alpha} A_{o} \kappa_{o \alpha \beta} \sin \delta_{o}
\end{array}\right] \\
& {\left[\begin{array}{c}
R_{\beta} \\
I_{\beta}
\end{array}\right] .}
\end{aligned}
$$

If we assume $\left[R_{\alpha}, I_{\alpha}\right] \propto e^{\text {st }} \forall \alpha$, then this equation becomes

$$
\begin{aligned}
& 0= \\
& {\left[\begin{array}{cc}
-\left(\gamma_{\alpha}+s\right)+\omega_{\alpha} A_{o} \kappa_{o \alpha \alpha} \sin \delta_{o} & \Delta_{\alpha}+\omega_{\alpha} A_{o} \kappa_{o \alpha \alpha} \cos \delta_{o} \\
-\Delta_{\alpha}+\omega_{\alpha} A_{o} \kappa_{o \alpha \alpha} \cos \delta_{o} & -\left(\gamma_{\alpha}+s\right)-\omega_{\alpha} A_{o} \kappa_{o \alpha \alpha} \sin \delta_{o}
\end{array}\right]} \\
& \times\left[\begin{array}{c}
R_{\alpha} \\
I_{\alpha}
\end{array}\right] \\
& +\sum_{\beta \neq \alpha}\left[\begin{array}{ll}
2 \omega_{\alpha} A_{o} \kappa_{o \alpha \beta} \sin \delta_{o} & 2 \omega_{\alpha} A_{o} \kappa_{o \alpha \beta} \cos \delta_{o} \\
2 \omega_{\alpha} A_{o} \kappa_{o \alpha \beta} \cos \delta_{o} & -2 \omega_{\alpha} A_{o} \kappa_{o \alpha \beta} \sin \delta_{o}
\end{array}\right]\left[\begin{array}{c}
R_{\beta} \\
I_{\beta}
\end{array}\right] \text {. }
\end{aligned}
$$

This is an eigenvalue problem for a large matrix and the general decomposition is difficult. However, the matrix can be made almost symmetric and if we make several approximations the problem becomes analytically tractable. Specifically, if we assume

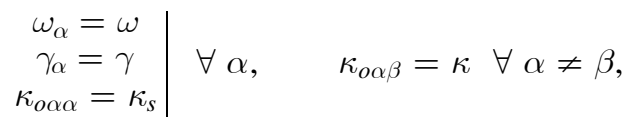

then we can define

$$
\begin{aligned}
M_{S} & \equiv\left[\begin{array}{ll}
-(\gamma+s)+\omega A_{o} \kappa_{s} \sin \delta_{o} & \Delta+\omega A_{o} \kappa_{s} \cos \delta_{o} \\
-\Delta+\omega A_{o} \kappa_{S} \cos \delta_{o} & -(\gamma+s)-\omega A_{o} \kappa_{S} \sin \delta_{o}
\end{array}\right], \\
M_{I} & \equiv\left[\begin{array}{ll}
2 \omega A_{o} \kappa \sin \delta_{o} & 2 \omega A_{o} \kappa \cos \delta_{o} \\
2 \omega A_{o} \kappa \cos \delta_{o} & -2 \omega A_{o} \kappa \sin \delta_{o}
\end{array}\right],
\end{aligned}
$$

where $\Delta_{\alpha}=\Delta \forall \alpha$ since $\omega_{\alpha}=\omega \forall \alpha$. Writing this as a single matrix and requiring non-trivial mode amplitudes, we obtain

$$
\begin{gathered}
0=\operatorname{det}\left|\begin{array}{ccccccc}
M_{S} & M_{I} & M_{I} & M_{I} & \cdots & M_{I} & M_{I} \\
M_{I} & M_{S} & M_{I} & M_{I} & \cdots & M_{I} & M_{I} \\
M_{I} & M_{I} & M_{S} & M_{I} & \cdots & M_{I} & M_{I} \\
M_{I} & M_{I} & M_{I} & M_{S} & \cdots & M_{I} & M_{I} \\
\vdots & \vdots & \vdots & \vdots & \ddots & \vdots & \vdots \\
M_{I} & M_{I} & M_{I} & M_{I} & \cdots & M_{S} & M_{I} \\
M_{I} & M_{I} & M_{I} & M_{I} & \cdots & M_{I} & M_{S}
\end{array}\right| \\
=\operatorname{det}\left|M_{S}-M_{I}\right|^{N-1} \operatorname{det}\left|M_{S}+(N-1) M_{I}\right| .
\end{gathered}
$$

We have $N-1$ repeated pairs of roots and one additional pair. The eigenvalues can be easily computed from

$$
\begin{gathered}
\operatorname{det}\left|M_{S}-M_{I}\right|=(\gamma+s)^{2}+\Delta^{2}-\omega^{2} A_{o}^{2}\left(\kappa_{s}-2 \kappa\right)^{2}=0 \\
\Rightarrow s=-\gamma \pm \sqrt{\omega^{2} A_{o}^{2}\left(2 \kappa-\kappa_{s}\right)^{2}-\Delta^{2}}
\end{gathered}
$$

and

$$
\begin{aligned}
& \text { det }\left|M_{S}+(N-1) M_{I}\right| \\
& =(\gamma+s)^{2}+\Delta^{2}-\omega^{2} A_{o}^{2}\left(2(N-1) \kappa+\kappa_{s}\right)^{2}=0 \\
& \Rightarrow s=-\gamma \pm \sqrt{\omega^{2} A_{o}^{2}\left(2(N-1) \kappa+\kappa_{s}\right)^{2}-\Delta^{2}}
\end{aligned}
$$


In particular, we are interested in the values of $A_{o}$ for which $\mathbb{R}\{s\} \rightarrow 0$. These are

$$
\begin{aligned}
A_{\mathrm{thr}}^{2} & =\frac{\gamma^{2}+\Delta^{2}}{4 \omega^{2}\left(\kappa-\frac{1}{2} \kappa_{s}\right)^{2}} \\
& =\frac{\gamma \gamma}{4 \omega \omega\left(\kappa-\frac{1}{2} \kappa_{s}\right)^{2}}\left[1+\frac{(\Delta+\Delta)^{2}}{(\gamma+\gamma)^{2}}\right]
\end{aligned}
$$

$$
\begin{aligned}
& 0=\operatorname{det} \\
& \begin{array}{|ccccccccccccccc|}
M_{S}^{A} & M_{I}^{A} & \cdots & M_{I}^{A} & M_{I}^{A} & M_{I}^{A} & M_{I}^{A} & \cdots & M_{I}^{A} & M_{I}^{A} & 0 & 0 & \cdots & 0 & 0 \\
M_{I}^{A} & M_{S}^{A} & \cdots & M_{I}^{A} & M_{I}^{A} & M_{I}^{A} & M_{I}^{A} & \cdots & M_{I}^{A} & M_{I}^{A} & 0 & 0 & \cdots & 0 & 0 \\
\vdots & \vdots & & \vdots & \vdots & \vdots & \vdots & & \vdots & \vdots & \vdots & \vdots & \vdots & \vdots & \vdots \\
M_{I}^{A} & M_{I}^{A} & \cdots & M_{S}^{A} & M_{I}^{A} & M_{I}^{A} & M_{I}^{A} & \cdots & M_{I}^{A} & M_{I}^{A} & 0 & 0 & \cdots & 0 & 0 \\
M_{I}^{A} & M_{I}^{A} & \cdots & M_{I}^{A} & M_{S}^{A} & M_{I}^{A} & M_{I}^{A} & \cdots & M_{I}^{A} & M_{I}^{A} & 0 & 0 & \cdots & 0 & 0 \\
M_{I}^{A} & M_{I}^{A} & \cdots & M_{I}^{A} & M_{I}^{A} & M_{S}^{C} & M_{I}^{C} & \cdots & M_{I}^{C} & M_{I}^{C} & M_{I}^{B} & M_{I}^{B} & \cdots & M_{I}^{B} & M_{I}^{B} \\
M_{I}^{A} & M_{I}^{A} & \cdots & M_{I}^{A} & M_{I}^{A} & M_{I}^{C} & M_{S}^{C} & \cdots & M_{I}^{C} & M_{I}^{C} & M_{I}^{B} & M_{I}^{B} & \cdots & M_{I}^{B} & M_{I}^{B} \\
\vdots & \vdots & & \vdots & \vdots & \vdots & \vdots & & \vdots & \vdots & \vdots & \vdots & & \vdots & \vdots \\
M_{I}^{A} & M_{I}^{A} & \cdots & M_{I}^{A} & M_{I}^{A} & M_{I}^{C} & M_{I}^{C} & \cdots & M_{S}^{C} & M_{I}^{C} & M_{I}^{B} & M_{I}^{B} & \cdots & M_{I}^{B} & M_{I}^{B} \\
M_{I}^{A} & M_{I}^{A} & \cdots & M_{I}^{A} & M_{I}^{A} & M_{I}^{C} & M_{I}^{C} & \cdots & M_{I}^{C} & M_{S}^{C} & M_{I}^{B} & M_{I}^{B} & \cdots & M_{I}^{B} & M_{I}^{B} \\
0 & 0 & \cdots & 0 & 0 & M_{I}^{B} & M_{I}^{B} & \cdots & M_{I}^{B} & M_{I}^{B} & M_{S}^{B} & M_{I}^{B} & \cdots & M_{I}^{B} & M_{I}^{B} \\
0 & 0 & \cdots & 0 & 0 & M_{I}^{B} & M_{I}^{B} & \cdots & M_{I}^{B} & M_{I}^{B} & M_{I}^{B} & M_{S}^{B} & \cdots & M_{I}^{B} & M_{I}^{B} \\
\vdots & \vdots & & \vdots & \vdots & \vdots & \vdots & & \vdots & \vdots & \vdots & \vdots & & \vdots & \vdots \\
0 & 0 & \cdots & 0 & 0 & M_{I}^{B} & M_{I}^{B} & \cdots & M_{I}^{B} & M_{I}^{B} & M_{I}^{B} & M_{I}^{B} & \cdots & M_{S}^{B} & M_{I}^{B} \\
0 & 0 & \cdots & 0 & 0 & M_{I}^{B} & M_{I}^{B} & \cdots & M_{I}^{B} & M_{I}^{B} & M_{I}^{B} & M_{I}^{B} & \cdots & M_{I}^{B} & M_{S}^{B}
\end{array}
\end{aligned}
$$

and

$$
\begin{aligned}
A_{\mathrm{thr}}^{2}= & \frac{\gamma^{2}+\Delta^{2}}{4 \omega^{2}\left((N-1) \kappa+\frac{1}{2} \kappa_{s}\right)^{2}} \\
= & \left(\frac{1}{N-1}\right)^{2} \frac{\gamma \gamma}{4 \omega \omega\left(\kappa+\frac{1}{2(N-1)} \kappa_{s}\right)^{2}} \\
& \times\left[1+\frac{(\Delta+\Delta)^{2}}{(\gamma+\gamma)^{2}}\right],
\end{aligned}
$$

respectively. We see that there are $N-1$ modes that resemble "standard" three-mode instabilities and one collective eigenvalue, with an amplitude threshold suppressed by a factor of $N-1$.
Because of the assumptions in Equation (60), the actual value of $A_{\text {thr }}$ will differ somewhat from this expression. Nonetheless, we expect it to generalize to the requirement that

$$
(N-1)^{2} A_{o}^{2} \gtrsim \frac{\gamma_{1} \gamma_{2}}{4 \omega_{1} \omega_{2} \kappa_{o 12}^{2}} \times\left[1+\frac{\left(\Delta_{1}+\Delta_{2}\right)^{2}}{\left(\gamma_{1}+\gamma_{2}\right)^{2}}\right]
$$

$\forall$ modes $1,2 \in$ collective set of $N$ modes,

where $\Delta_{1}+\Delta_{2}=\Omega+\omega_{1}+\omega_{2}$.

\section{E.2. Overlapping Collective Modes Stability}

We now consider a coupling topology where there are three types of modes. The $A$ modes are coupled to other $A$ modes and to $C$ modes. $B$ modes are coupled to other $B$ modes and to $C$ modes. $C$ modes are coupled to all other modes. Furthermore, we assume that all $A, B$, and $C$ modes are coupled to the same parent modes, which we treat as a single parent even though multiple parents may be acting (see Appendix C).

The associated eigenvalue problem yields the following characteristic equation
We again note the high degree of symmetry, which allows us reduce the determinant to

$$
\begin{aligned}
0= & \left(\operatorname{det}\left|M_{S}^{A}-M_{I}^{A}\right|\right)^{N_{a}-1}\left(\operatorname{det}\left|M_{S}^{B}-M_{I}^{B}\right|\right)^{N_{b}-1} \\
& \times\left(\operatorname{det}\left|M_{S}^{C}-M_{I}^{C}\right|\right)^{N_{c}-1} \\
& \times \operatorname{det}\left|M_{S}^{A}+\left(N_{a}-1\right) M_{I}^{A}\right| \operatorname{det}\left|M_{S}^{B}+\left(N_{b}-1\right) M_{I}^{B}\right| \\
& \times \operatorname{det}\left|M_{S}^{C}+\left(N_{c}-1\right) M_{I}^{C}-N_{c} Z\right|,
\end{aligned}
$$

where

$$
\begin{aligned}
Z= & N_{a} M_{I}^{A}\left(M_{S}^{A}+\left(N_{a}-1\right) M_{I}^{A}\right)^{-1} M_{I}^{A} \\
& +N_{b} M_{I}^{b}\left(M_{S}^{B}+\left(N_{b}-1\right) M_{I}^{B}\right)^{-1} M_{I}^{B} .
\end{aligned}
$$


We recognize this as $N_{a}-1$ independent $A$ eigenvalues, $N_{b}-1$ independent $B$ eigenvalues, $N_{c}-1$ independent $C$ eigenvalues, one eigenvalue corresponding to the collective modes without the coupling to $C$ modes for each of the $A$ and $B$ modes, and a collective set for the $C$ modes with a modification due to the couplings to the $A$ and $B$ modes (through $Z$ ). We further note that when $N_{c} \rightarrow 0$, the eigenvalues reduce to two separate collective sets, as expected.

The interesting eigenvalue is due to the interaction between the $C$ modes' collective set and the couplings to $A$ and $B$ modes. If we assume that all mode parameters are the same for all sets of modes, and further assume that $N_{a}=N_{b}=N_{c}$, we can make analytic progress on this determinant, and obtain

$$
(\gamma+s)^{2}+\Delta^{2}-\omega^{2} A_{0}^{2}\left(\left(k_{s}+2(N-1) k\right)^{2}+8 N^{2} k^{2}\right)=0
$$

and the threshold amplitude

$$
\begin{aligned}
& A_{\mathrm{thr}}^{2}=\frac{\gamma^{2}+\Delta^{2}}{4 \omega^{2}\left(3 k^{2} N^{2}+k\left(k_{s}-2 k\right) N+k\left(k-k_{s}\right)+k_{s}^{2} / 4\right)} \\
& \approx \frac{1}{3 N^{2}}\left(\frac{\gamma^{2}+\Delta^{2}}{4 \omega^{2} k^{2}}\right)=\frac{1}{N_{a}^{2}+N_{b}^{2}+N_{c}^{2}}\left(\frac{\gamma^{2}+\Delta^{2}}{4 \omega^{2} k^{2}}\right),
\end{aligned}
$$

where we assumed the limit of large $N$. We note that this is very similar to the case of a single collective set, except $N^{2} \rightarrow N_{a}^{2}+N_{b}^{2}+N_{c}^{2}$. If we stitch together many separate collective sets by overlapping them, we only expect the effective number of modes to sum in quadrature. This was tested numerically by taking the determinant without assuming equal numbers of modes, and found to be in reasonable agreement with this scaling.

\section{E.3. Non- "Self Coupled" Collective Sets}

Appendices E.1 and E.2 considered self-coupled modes. However, the vast majority of couplings will be between modes that do not support self-coupled daughters. For example, if the parent azimuthal order $m$ is odd, then the daughter modes must have different $m$ numbers. If we consider two sets of modes, one with $N$ daughters and one with $n$ daughters, we can define $2 \times 2$ sub-matrices similar to Appendix E.1 for each group of modes. This means we will also find collective sets with characteristic equations like the following, with capital letters corresponding to the $N$-mode set and lower case letters corresponding to the $n$-mode set

$$
\left|\begin{array}{cccccccccc}
M_{S} & 0 & \cdots & 0 & 0 & M_{I} & M_{I} & \cdots & M_{I} & M_{I} \\
0 & M_{S} & \cdots & 0 & 0 & M_{I} & M_{I} & \cdots & M_{I} & M_{I} \\
\vdots & \vdots & & \vdots & \vdots & \vdots & \vdots & & \vdots & \vdots \\
0 & 0 & \cdots & M_{S} & 0 & M_{I} & M_{I} & \cdots & M_{I} & M_{I} \\
0 & 0 & \cdots & 0 & M_{S} & M_{I} & M_{I} & \cdots & M_{I} & M_{I} \\
M_{i} & M_{i} & \cdots & M_{i} & M_{i} & M_{s} & 0 & \cdots & 0 & 0 \\
M_{i} & M_{i} & \cdots & M_{i} & M_{i} & 0 & M_{s} & \cdots & 0 & 0 \\
\vdots & \vdots & & \vdots & \vdots & \vdots & \vdots & & \vdots & \vdots \\
M_{i} & M_{i} & \cdots & M_{i} & M_{i} & 0 & 0 & \cdots & M_{s} & 0 \\
M_{i} & M_{i} & \cdots & M_{i} & M_{i} & 0 & 0 & \cdots & 0 & M_{s}
\end{array}\right|
$$

where this is an $(N+n) \times(N+n)$ matrix. We can simplify this to only a $4 \times 4$ determinant

$$
0=\left(\operatorname{det}\left|M_{s}\right|\right)^{N-1}\left(\operatorname{det}\left|M_{s}\right|\right)^{n-1} \operatorname{det}\left|\begin{array}{cc}
M_{S} & n M_{I} \\
N M_{i} & M_{s}
\end{array}\right|,
$$

which looks like a set of independent eigenmodes and a $4 \times 4$ determinant for the collective modes. In general, that $4 \times 4$ determinant must be solved numerically. However, if we again assume identical mode parameters and that $N=n$, we see that this reduces to

$$
\operatorname{det}\left|\begin{array}{cc}
M_{S} & N M_{I} \\
N M_{I} & M_{S}
\end{array}\right|
$$

which looks just like the three-mode instability equations with $k \rightarrow N k$. Therefore, we can read off the amplitude threshold immediately. Again, we see that the threshold is decreased by a factor of $N$ compared to the three-mode threshold. We expect the threshold energy to approximately scale as

$$
A_{\mathrm{thr}}^{2} \approx\left(\frac{1}{N n}\right) \frac{\gamma^{2}+\Delta^{2}}{4 \omega^{2} k^{2}} .
$$

\section{E.4. Decoupling of "Very Different" Modes from Collective Sets}

In general, since all the mode parameters will be slightly different, our previous examples are a bit artificial. We now investigate the behavior when one mode begins to differ from the others. Consider the following characteristic equation, with $N$ identical modes and one slightly different mode indicated by $\delta M$

$$
0=\operatorname{det}\left|\begin{array}{ccccc}
M_{S} & M_{I} & \cdots & M_{I} & M_{I} \\
M_{I} & M_{S} & \cdots & M_{I} & M_{I} \\
\vdots & \vdots & & \vdots & \vdots \\
M_{I} & M_{I} & \cdots & M_{S} & M_{I} \\
M_{I} & M_{I} & \cdots & M_{I} & M_{S}+\delta M
\end{array}\right| \text {. }
$$

We can reduce this to

$$
\begin{aligned}
0= & \left(\operatorname{det}\left|M_{S}-M_{I}\right|\right)^{N-1} \operatorname{det}\left|\begin{array}{cc}
M_{S}-M_{I} & -\delta M \\
N M_{I} & M_{S}+\delta M
\end{array}\right| \\
= & \left(\operatorname{det}\left|M_{S}-M_{I}\right|\right)^{N-1} \operatorname{det}\left|M_{S}+(N-1) M_{I}\right| \operatorname{det} \\
& \times\left|M_{S}-N M_{I}\left(M_{S}+(N-1) M_{I}\right)^{-1} M_{I}+\delta M\right| .
\end{aligned}
$$

As $\delta M \rightarrow 0$, this reduces to a single collective set with $N \rightarrow N+1$, as expected. We also note that this looks like the eigenvalues of a normal collective set with $N$ modes and a new eigenvalue related to the different mode. Furthermore, if $\delta M$ dominates the new eigenvalue, then we see that the different mode will "decouple" from the other modes. Clearly, there will be some threshold for how large $\delta M$ needs to be before the different mode decouples, and that threshold will depend on the parent's amplitude in a non-trivial way. We expect that a large parent amplitude $A_{o}$ will support a larger $\delta M$ before the mode decouples. 


\section{APPENDIX F \\ SCALING OF PARAMETRIC INSTABILITY THRESHOLD AND WAVE BREAKING THRESHOLD}

Our calculations treat the system of modes as a set of global standing waves. However, if a wave's nonlinearity parameter $k_{r} \xi_{r} \gtrsim 1$, the wave will invert the stratification of the star and break (Goodman \& Dickson 1998; Barker \& Ogilvie 2010). Because it does not reflect at turning points within the propagation cavity, such a wave is more appropriately treated as a traveling wave rather than a standing wave. Given that we specifically focus on parent waves below the wave breaking threshold $\left(k_{r} \xi_{r} \lesssim 1\right)$, we know that the parent is well described as a standing wave. Here we are interested in determining whether the same is true of the daughters, granddaughters, etc.

As we describe in Section 3.1, the parametric instability threshold scales as $E_{\mathrm{thr}} \propto \omega^{6}$. This implies that each successive generation has a lower $E_{\mathrm{thr}}$ and is therefore ever more susceptible to parametric instabilities. We show below that the energy above which a wave breaks also scales as $E_{\text {break }} \propto \omega^{6}$. Moreover, we find that $E_{\text {thr }} \ll E_{\text {break. This means }}$ that well before the daughters, granddaughters, etc. reach the wave breaking limit $k_{r} \xi_{r} \gtrsim 1$, they will excite the next generation of modes through parametric instabilities. Although a mode is not necessarily limited to remain below its $E_{\mathrm{thr}}$, we do not expect it to greatly exceed it either. This is because as a mode's amplitude increases past its $E_{\mathrm{thr}}$, its children grow at an ever faster rate and thereby limit how far their parent overshoots $E_{\text {thr. }}$. While this issue requires further study, it suggests that our assumption that the modes are all global standing waves may be reasonable.

We begin by calculating $E_{\mathrm{thr}}$. For typical parameter values of a hot Jupiter system, $E_{\mathrm{thr}}$ is limited by the nonlinear detuning of the daughter modes rather than their linear damping (and similarly for granddaughters, etc.). To a first approximation, the detuning $\Delta$ is determined by half the frequency spacing between the daughter modes $\omega / 2 n$. However, this assumes that the lowest $E_{\text {thr }}$ pairs are self-coupled modes. Because there is a distribution of mode frequencies slightly above and below half the parent frequency, there are always some mode pairs that happen to have $\Delta \ll \omega / 2 n$ (Wu \& Goldreich 2001). These are the pairs that minimize $E_{\text {thr }}$. Writing $\Delta=\alpha \omega / 2 n$, where $\alpha \ll 1$ and using the expressions for $\omega, \gamma$, and $\kappa$ given in Section 2.2, we find that the threshold energy for self-coupled daughters is

$$
E_{\mathrm{thr}} \simeq 8 \times 10^{-16}\left(\frac{\alpha}{0.01}\right)^{2}\left(\frac{P}{\text { day }}\right)^{-6} E_{0},
$$

where $\alpha \sim 0.01$ based on our three-mode network search results (cf. Figure 1).

Now consider $k_{r} \xi_{r}$. It is at its maximum near the inner turning point of the parent (where $\omega \simeq N$ ). This is because in the core of a solar model, $k_{r} \simeq \Lambda N / \omega r$ is approximately constant and $\xi_{r} \propto r^{-2}$ by flux conservation. Using the WKB relations given in Appendix A of WAQB (see also Goodman \& Dickson 1998; Ogilvie \& Lin 2007), we find that the wave breaking condition $\max \left\{k_{r} \xi_{r}\right\}=1$ for $l=2$ modes corresponds to an energy

$$
E_{\text {brk }} \simeq 3 \times 10^{-13}\left(\frac{P}{\text { day }}\right)^{-6} E_{0} .
$$

Longer period modes break at lower amplitudes because they reach further into the core of the star. We thus see that both energies scale as $\omega^{6}$ and $E_{\mathrm{thr}} \ll E_{\mathrm{brk}}$, as claimed.

\section{APPENDIX G \\ ESTIMATE OF THE LINEAR AND PARENT-DAUGHTER ORBITAL DECAY TIMESCALES}

The linear dissipation rate of individual resonant modes is $\dot{E}_{\text {lin }} \simeq 2 \gamma_{\alpha} E_{\text {lin }}$, where $E_{\text {lin }}$ is given by Equation (15). For the short periods that we consider, $\Delta_{\alpha} \approx \omega_{\alpha} / 2 n_{\alpha} \gg \gamma_{\alpha}$. After summing over many parents near the resonance, using the WKB estimates for the damping and forcing coefficients (Equations (8b) and (9)), and averaging according to Appendix A, we find

$$
\begin{aligned}
& \left\langle\tau_{E}\right\rangle_{\text {lin }} \\
& \simeq 1.4 \times 10^{12}\left(\frac{M}{M_{\odot}}\right)^{-5 / 6}\left(\frac{R}{R_{\odot}}\right)^{11 / 2}\left(\frac{M_{p}}{M_{\mathrm{J}}}\right)^{-1}\left(\frac{P}{\text { day }}\right)^{3} \text { year }
\end{aligned}
$$

and

$$
Q_{*, \text { lin }}^{\prime} \simeq 1.1 \times 10^{10}\left(\frac{R}{R_{\odot}}\right)^{21 / 2}\left(\frac{M}{M_{\odot}}\right)^{-27 / 6}\left(\frac{M_{p}}{M_{\mathrm{J}}}\right)\left(\frac{P}{\mathrm{day}}\right)^{-4 / 3}
$$

As we explain in Section 4.2, we can also estimate the nonlinear dissipation rate of networks consisting of only parents and daughters (but not granddaughters, etc.). This is because the dissipation in that case is dominated by the single daughter pair $(\beta, \gamma)$ with the lowest instability threshold $E_{\mathrm{thr}}$. As we show in Appendix B, for the parameters of a hot Jupiter system, the nonlinear equilibrium energy of such a daughter pair is $E_{\beta, \gamma} \simeq\left|U_{\alpha} / 2 \kappa_{\alpha \beta \gamma}\right| E_{0}$. The total dissipation rate of the system is approximately the dissipation due to these two daughters $\dot{E}_{\mathrm{p}-\mathrm{d}} \simeq 2 \times 2 \gamma_{\beta, \gamma} E_{\beta, \gamma}$. There is a small correction to this because the lowest $E_{\text {thr }}$ daughters have slightly different parameters and therefore do not sit at exactly the same amplitudes. After accounting for this small correction and plugging in Equations (8b), (9) and (10), we find

$$
\begin{aligned}
\left\langle\tau_{E}\right\rangle_{\mathrm{p}-\mathrm{d}} \simeq & 2.0 \times 10^{11}\left(\frac{\Lambda_{\beta, \gamma}^{2}}{2}\right)^{-1}\left(\frac{T_{\alpha \beta \gamma}}{0.2}\right)\left(\frac{M}{M_{\odot}}\right)^{-11 / 6} \\
& \times\left(\frac{R}{R_{\odot}}\right)^{11 / 2}\left(\frac{P}{\text { day }}\right)^{19 / 6} \text { year }
\end{aligned}
$$

and

$$
\begin{aligned}
Q_{*, \mathrm{p}-\mathrm{d}}^{\prime} \simeq & 1.5 \times 10^{9}\left(\frac{\Lambda_{\beta, \gamma}^{2}}{2}\right)^{-1}\left(\frac{T_{\alpha \beta \gamma}}{0.2}\right)\left(\frac{M}{M_{\odot}}\right)^{-27 / 6} \\
& \times\left(\frac{R}{R_{\odot}}\right)^{21 / 2}\left(\frac{M_{p}}{M_{\mathrm{J}}}\right)\left(\frac{P}{\text { day }}\right)^{-7 / 6} \cdot
\end{aligned}
$$

Here we took $l_{\beta, \gamma}=1$, which is representative of the typical lowest $E_{\text {thr }}$ daughters for $P \gtrsim 2$ days. We find good agreement between the parent-daughter network integrations that include many daughters and this analytic estimate (see circles and dashed curve in Figure 10). In the figure, we assume $l_{\beta, \gamma}=1$ 
even for $P<2$ days. However, at these shorter periods, the available daughter modes are spaced further apart in frequency and the lowest $E_{\text {thr }}$ pair may be pushed to $l_{\beta, \gamma} \gtrsim 1$. This causes the small discrepancy between the circles and dashed curve at $P \lesssim 2$ days seen in Figure 10 .

\section{REFEREN.CES}

Aerts, C., Christensen-Dalsgaard, J., \& Kurtz, D. 2010, Asteroseismology (Berlin: Springer)

Alonso, R., Auvergne, M., Baglin, A., et al. 2008, A\&A, 482, L21

Bakos, G. Á., Hartman, J. D., Torres, G., et al. 2012, AJ, 144, 19

Barker, A. J. 2011, MNRAS, 414, 1365

Barker, A. J., \& Lithwick, Y. 2014, MNRAS, 437, 305

Barker, A. J., \& Ogilvie, G. I. 2010, MNRAS, 404, 1849

Barker, A. J., \& Ogilvie, G. I. 2011, MNRAS, 417, 745

Birkby, J. L., Cappetta, M., Cruz, P., et al. 2014, MNRAS, 440, 1470

Bondarescu, R., Teukolsky, S. A., \& Wasserman, I. 2009, PhRvD, 79, 104003

Brink, J., Teukolsky, S. A., \& Wasserman, I. 2005, PhRvD, 71, 064029

Gandolfi, D., Parviainen, H., Deeg, H. J., et al. 2015, A\&A, 576, A11

Gillon, M., Lanotte, A. A., Barman, T., et al. 2010, A\&A, 511, A3

Gillon, M., Smalley, B., Hebb, L., et al. 2009, A\&A, 496, 259

Goldreich, P., \& Soter, S. 1966, Icar, 5, 375

Goodman, J., \& Dickson, E. S. 1998, ApJ, 507, 938

Hebb, L., Collier-Cameron, A., Triaud, A. H. M. J., et al. 2010, ApJ, 708, 224
Hellier, C., Anderson, D. R., Collier-Cameron, A., et al. 2011, ApJL, 730, L31 Jackson, B., Barnes, R., \& Greenberg, R. 2009, ApJ, 698, 1357

Jackson, B., Greenberg, R., \& Barnes, R. 2008, ApJ, 678, 1396

Kumar, P., \& Goodman, J. 1996, ApJ, 466, 946

McQuillan, A., Mazeh, T., \& Aigrain, S. 2013, ApJL, 775, L11

Meibom, S., \& Mathieu, R. D. 2005, ApJ, 620, 970

Mortier, A., Santos, N. C., Sousa, S. G., et al. 2013, A\&A, 558, A106

Ogilvie, G. I. 2014, ARA\&A, 52, 171

Ogilvie, G. I., \& Lin, D. N. C. 2007, ApJ, 661, 1180

O'Leary, R. M., \& Burkart, J. 2014, MNRAS, 440, 3036

Paxton, B. 2004, PASP, 116, 699

Penev, K., Jackson, B., Spada, F., \& Thom, N. 2012, ApJ, 751, 96

Penev, K., \& Sasselov, D. 2011, ApJ, 731, 67

Schenk, A. K., Arras, P., Flanagan, E. E., Teukolsky, S. A., \& Wasserman, I. 2001, PhRvD, 65, 024001

Smith, A. M. S., Anderson, D. R., Collier Cameron, A., et al. 2012, AJ, 143, 81

Storch, N. I., \& Lai, D. 2014, MNRAS, 438, 1526

Teitler, S., \& Königl, A. 2014, ApJ, 786, 139

Terquem, C., Papaloizou, J. C. B., Nelson, R. P., \& Lin, D. N. C. 1998, ApJ, 502,788

Udry, S., \& Santos, N. C. 2007, ARA\&A, 45, 397

Valsecchi, F., \& Rasio, F. A. 2014, ApJL, 787, L9

Van Hoolst, T. 1994, A\&A, 286, 879

Venumadhav, T., Zimmerman, A., \& Hirata, C. M. 2014, ApJ, 781, 23

Watson, C. A., \& Marsh, T. R. 2010, MNRAS, 405, 2037

Weinberg, N. N., Arras, P., Quataert, E., \& Burkart, J. 2012, ApJ, 751, 136

Winn, J. N., \& Fabrycky, D. C. 2015, ARA\&A, 53, 409

Wu, Y., \& Goldreich, P. 2001, ApJ, 546, 469 